\title{
Multiparticle Localization at Low Energy for Multidimensional Continuous Anderson Models
}

\author{
Trésor Ekanga \\ Université Paris Diderot, 13 Rue Albert Einstein, 75013 Paris, France \\ Correspondence should be addressed to Trésor Ekanga; ekanga@math.cnrs.fr
}

Received 28 April 2019; Revised 21 August 2020; Accepted 6 September 2019; Published 4 May 2020

Academic Editor: Laurent Raymond

Copyright (c) 2020 Trésor Ekanga. This is an open access article distributed under the Creative Commons Attribution License, which permits unrestricted use, distribution, and reproduction in any medium, provided the original work is properly cited.

\begin{abstract}
We study the multiparticle Anderson model in the continuum and show that under some mild assumptions on the random external potential and the inter-particle interaction, for any finite number of particles, the multiparticle lower spectral edges are almost surely constant in absence of ergodicity. We stress that this result is not quite obvious and has to be handled carefully. In addition, we prove the spectral exponential and the strong dynamical localization of the continuous multiparticle Anderson model at low energy. The proof based on the multiparticle multiscale analysis bounds needs the values of the external random potential to be independent and identically distributed, whose common probability distribution is at least Log-Hölder continuous.
\end{abstract}

\section{Introduction}

This paper follows our previous works $[1,2]$ on localization for multiparticle random lattice Schrödinger operators at low energy. Some other papers [3-10] analyzed multiparticle models in the regime including the strong disorder or the low energy and for different type of models such as the alloy-type Anderson model or the multiparticle Anderson model in quantum graphs [11].

In their work [10], Klein and Nguyen developed the continuum multiparticle bootstrap multiscale analysis of the Anderson model with alloy type external potential. The method of Klein and Nguyen is very close in the spirit to that of our work [2]. The results of [2] were the first rigorous mathematical proof of localization for many body interacting Hamiltonians near the bottom of the spectrum on the lattice. In the present paper we prove similar results in the continuum.

The work by Sabri [11], uses a different strategy in the course of the multiparticle multiscale analysis at low energy. The analysis is made by considering the Green functions, i.e., the matrix elements of the local resolvent operator instead of the norm of the kernel as it will be developed in this paper and this obliged the author to modify the standard Combes Thomas estimate and adapted it to matrix elements of the local resolvent. Also, our proof on the almost surely spectrum is completely different. The scale induction step in the multiparticle multiscale analysis as well as the strategy of the localization proofs is also different. Chulaevsky [6] used the results of Klein and Nguyen [10] and analyzed multiparticle random operators with alloy-type external potential with infinite range interaction at low energy.

Let us emphasize that the almost sure nonrandomness of the bottom of the spectrum of the multiparticle random Hamiltonian is the heart the problem of localization at low energy for multiparticle systems. In this work, we propose a very clear and constructive proof of this fact. We also prove the exponential localization in the max-norm and the strong dynamical localization near the bottom of the spectrum.

Our multiparticle multiscale analysis is more close in the spirit to its single particle counterpart developed by Stollmann [12] in the continuum case and by von Dreifus and Klein [13] in the lattice case.

Let us now discuss on the structure of the paper. In the next Section, we set up the model, give the assumptions and formulate the main results. In Section 3, we give two important results for our multiparticle multiscale analysis scheme, namely, the Wegner and the Combes Thomas estimates, one, important to bound the probability of resonances, while the other is used to bound the initial scale lengths estimates for energies near the bottom of the spectrum. In Section 4, we prove the initial length scale of the multiscale analysis. Section 
5 is devoted the multiparticle multiscale induction step. In Section 6 we prove the variable energy multiparticle multiscale analysis result. Finally, in Section 7, We prove the main results.

\section{The Model Assumptions and the Main Results}

2.1. The Model. We fix at the very beginning the number of the particles $N \geq 2$. We are concerned with multiparticle random Schrödinger operators of the forms:

$$
\mathbf{H}^{(N)}(\omega):=-\Delta+\mathbf{U}+\mathbf{V},
$$

acting in $L^{2}\left(\left(\mathbb{R}^{d}\right)^{N}\right)$. Sometimes, we will use the identification $\left(\mathbb{R}^{d}\right) N \cong \mathbb{R}^{N d}$. Above, $\boldsymbol{\Delta}$ is the Laplacian on $\mathbb{R}^{N d}, \mathbf{U}$ represents the inter-particle interaction potential which acts as a multiplication operator in $L^{2}\left(\mathbb{R}^{N d}\right)$. Additional information in $\mathbf{U}$ is given in the assumptions. $\mathbf{V}$ is the multiparticle random external potential, also acting as multiplication operator on $L^{2}\left(\mathbb{R}^{N d}\right)$. For $\mathbf{x}=\left(x_{1}, \ldots, x_{N}\right) \in\left(\mathbb{R}^{d}\right)^{N}$, $\mathbf{V}(\mathbf{x})=V\left(x_{1}\right)+\cdots+V\left(x_{N}\right)$ and $\left\{V(x, \omega), x \in \mathbb{Z}^{d}\right\}$ is an i.i.d. random stochastic process relative to the probability space $(\Omega, \mathscr{B}, \mathbb{P})$ with $\Omega=\mathbb{R}^{\mathbb{Z}^{d}}, \mathscr{B}=\otimes_{x \in \mathbb{Z}^{d}} B(\mathbb{R})$ and $\mathbb{P}=\otimes_{x \in \mathbb{Z}^{d}} \mu$ where $\mu$ is the common probability measure of the i.i.d. random variables $\left\{V(x, \omega): x \in \mathbb{Z}^{d}\right\}$. Explicitly, we have that for

$$
\begin{aligned}
& \text { any } \boldsymbol{\Psi} \in L^{2}\left(\left(\mathbb{R}^{d}\right)^{N}\right) \\
& \begin{aligned}
(\mathbf{V} \boldsymbol{\Psi})(\mathbf{x}) & :=\mathbf{V}(\mathbf{x}) \boldsymbol{\Psi}(\mathbf{x})=\left(V\left(x_{1}, \omega\right)+\cdots+V\left(x_{N}, \omega\right)\right) \boldsymbol{\Psi}(\mathbf{x}), \\
\quad \mathbf{x} & =\left(x_{1}, \ldots, x_{N}\right) \in\left(\mathbb{Z}^{d}\right)^{N} .
\end{aligned}
\end{aligned}
$$

Observe that the noninteracting Hamiltonian $\mathbf{H}_{0}^{(N)}(\omega)$ can be written as a tensor product

$$
\mathbf{H}_{0}^{(N)}(\omega):=-\Delta+\mathbf{V}=\sum_{k=1}^{N} \mathbf{1}_{L^{2}\left(\mathbb{R}^{d}\right)}^{\otimes(k-1)} \otimes H^{(1)}(\omega) \otimes 1_{L^{2}\left(\mathbb{R}^{d}\right)}^{(N-k)}
$$

where, $H^{(1)}(\omega)=-\Delta+V(x, \omega)$ acting on $L^{2}\left(\mathbb{R}^{d}\right)$. We will also consider random Hamiltonian $\mathbf{H}^{(n)}(\omega) ; n=1, \ldots, N$ defined similarly. Denote by $|\cdot|$ the max-norm in $\mathbb{R}^{\text {nd }}$.

\subsection{Assumptions.}

(I) Short-Range Interaction. Fix any $n=1, \ldots, N$. The potential of inter-particle interaction $\mathbf{U}$ is bounded, nonnegative and of the form

$$
\mathbf{U}(\mathbf{x})=\sum_{1 \leq i \leq j \leq n ; i \neq j} \Phi\left(\left|x_{i}-x_{j}\right|\right), \quad \mathbf{x}=\left(x_{1}, \ldots, x_{n}\right),
$$

where $\Phi: \mathbb{R}_{+} \rightarrow \mathbb{R}$ is a compactly supported function such that

$$
\exists r_{0} \in \mathbb{N}: \operatorname{supp} \Phi \subset\left[0, r_{0}\right] .
$$

The external random potential $V: \mathbb{Z}^{d} \times \Omega \rightarrow \mathbb{R}$ is an i.i.d. random field relative to $(\Omega, \mathscr{B}, \mathbb{P})$ and is defined by

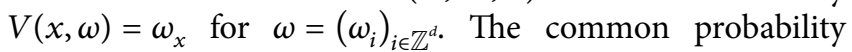

distribution function, $F_{V}$, of the i.i.d. random variables $V(x, \cdot), x \in \mathbb{Z}^{d}$ associated to the measure $\mu$ is defined by:

$$
F_{V}: t \rightarrow \mathbb{P}\{V(0, \omega) \leq t\}
$$

(P) Log-Hölder Continuity Condition. The random potential field $\left\{V(x, \omega) ; x \in \mathbb{Z}^{d}\right\}$ is i.i.d., of nonnegative values and the corresponding probability distribution function $F_{V}$ is $\log$ Hölder continuous: more precisely,

$$
s\left(F_{V}, \varepsilon\right):=\sup _{a \in \mathbb{R}}\left(F_{V}(a+\varepsilon)-F_{V}(a)\right) \leq \frac{C}{|\ln \varepsilon|^{2 A}}
$$

for some $C \in(0,+\infty)$ and $A \geq \frac{3}{2} \times 4^{N} p+9 N d$.

Note that this last condition depends on the parameter $p$ which will be introduced in Section 3 .

2.3. The Results. For any $n=1, \ldots, N$ we denote by $\sigma\left(\mathbf{H}^{(n)}(\omega)\right)$ the spectrum of $\mathbf{H}^{(n)}(\omega)$ and $E_{0}^{(n)}(\omega)$ the infimum of $\sigma\left(\mathbf{H}^{(n)}(\omega)\right)$.

Theorem 1. Let $1 \leq n \leq N$. Under assumptions (I) and (P) we have with probability one:

$$
\sigma\left(\mathbf{H}^{(n)}(\omega)\right)=[0, \infty)
$$

Consequently,

$$
\inf \sigma(\mathbf{H}(\omega))=0 \quad \text { a.s. }
$$

Theorem 2. Under the assumptions (I) and (P), there exists $E^{*}$ bigger than $E_{0}^{(N)}$ such that with $\mathbb{P}$-probability one:

(i) the spectrum of $\mathbf{H}^{(N)}(\omega)$ in $\left[E_{0}^{(N)}, E^{*}\right]$ is nonempty and pure point,

(ii) any eigenfuction corresponding to eigenvalues in $\left[E_{0}^{(N)}, E^{*}\right]$ is exponentially decaying at infinity in the max-norm.

Theorem 3. Assume that the hypotheses (I) and (P) hold true, then there exists $E^{*}$ bigger than $E_{0}^{(N)}$ and a positive $s^{*}(N, d)$ such that for any bounded $\mathbf{K} \subset \mathbb{Z}^{\text {nd }}$ and any $s \in\left(0, s^{*}\right)$ we have

$$
\mathbb{E}\left[\sup _{t \geq 0}\left\||\mathbf{X}|^{(s / 2)} \mathrm{e}^{-i t \mathbf{H}^{(N)}(\omega)} \mathbf{P}_{I}\left(\mathbf{H}^{(N)}(\omega)\right) \mathbf{1}_{\mathbf{K}}\right\|_{L^{2}\left(\mathbb{R}^{N d}\right)}\right]
$$

is finite, where $(|\mathbf{X}| \Psi)(\mathbf{x}):=|\mathbf{x}| \Psi(\mathbf{x}), \mathbf{P}_{I}\left(\mathbf{H}^{(N)}(\omega)\right)$ is the spectral projection of $\mathbf{H}^{(N)}(\omega)$ onto the interval $I:=\left[E_{0}^{(N)}, E^{*}\right]$ and $\mathbf{K} \subset \mathbb{R}$ is a compact domain.

Some parts of the rest of the text overlap with the paper [14] but for the reader convenience we give all the details of the arguments.

\section{Input for the Multiparticle Multiscale Analysis and Geometry}

3.1. Geometric Facts. According to the general structure of the multiscale analysis, we work with rectangular domains. For $\mathbf{u}=\left(u_{1}, \ldots, u_{n}\right) \in \mathbb{Z}^{n d}$, we denote by $\mathbf{C}_{L}^{(n)}(\mathbf{u})$ the $n$-particle cube, i.e.,

$$
\mathbf{C}_{L}^{(n)}=\left\{\mathbf{x} \in \mathbb{R}^{n d}:|\mathbf{x}-\mathbf{u}| \leq L\right\}
$$


and given $\left\{L_{i}: i=1, \ldots, n\right\}$, we define the rectangle

$$
\mathbf{C}^{(n)}(\mathbf{u})=\prod_{i=1}^{n} C_{L_{i}}^{(1)}\left(u_{i}\right)
$$

where $C_{L_{i}}^{(1)}\left(u_{i}\right)$ are the cubes of side length $2 L_{i}$, center at points $u_{i} \in \mathbb{Z}^{d}$. We also define

$\mathbf{C}_{L}^{(n, \text { int })}(\mathbf{u}):=\mathbf{C}_{L / 3}^{(n)}(\mathbf{u}), \quad \mathbf{C}_{L}^{(n, \text { out })}(\mathbf{u}):=\mathbf{C}_{L}^{(n)}(\mathbf{u}) \backslash \mathbf{C}_{L-2}^{(n)}(\mathbf{u}), \quad \mathbf{u} \in \mathbb{Z}^{\text {nd }}$,

and introduce the characteristic functions:

$$
\mathbf{1}_{\mathbf{x}}^{(n, \text { int })}:=\mathbf{1}_{C_{L}^{(n, \text { int })}(\mathbf{x})}, \quad \mathbf{1}_{\mathbf{x}}^{(n, \text { out })}:=\mathbf{1}_{C_{L}^{(n, \text { out })}(\mathbf{x})^{*}}
$$

The volume of the cube $\mathbf{C}_{L}^{(n)}(\mathbf{u})$ is $\left|\mathbf{C}_{L}^{(n)}(\mathbf{u})\right|=(2 L)^{n d}$. We denote the restriction of the Hamiltonian $\mathbf{H}^{(n)}$ to $\mathbf{C}^{(n)}(\mathbf{u})$ by

$$
\mathbf{H}_{\mathbf{C}^{(n)}(\mathbf{u})}^{(n)}=\left.\mathbf{H}^{(n)}\right|_{\mathbf{C}^{(n)}(\mathbf{u})}
$$

with dirichlet boundary conditions.

We denote the spectrum of $\mathbf{H}_{\mathbf{C}^{(n)}(\mathbf{u})}^{(n)}$ by $\sigma\left(\mathbf{H}_{\mathbf{C}^{(n)}(\mathbf{u})}^{(n)}\right)$ and its resolvent by

$\mathbf{G}_{\mathbf{C}^{(n)}(\mathbf{u})}^{(n)}(E):=\left(\mathbf{H}_{\mathbf{C}^{(n)}(\mathbf{u})}^{(n)}-E\right)^{-1}, \quad E \in \mathbb{R} \backslash \sigma\left(\mathbf{H}_{\mathbf{C}^{(n)}(\mathbf{u})}^{(n)}\right)$.

Let $m$ be a positive constant and consider $E \in \mathbb{R}$. A cube $\mathbf{C}_{L}^{(n)}(\mathbf{u}) \subset \mathbb{R}^{n d}, 1 \leq n \leq N$ will be called $(E, m)$-nonsingular $((E, m)-\mathrm{NS})$ if $E \notin \sigma\left(\mathbf{H}_{\mathbf{C}_{L}^{(n)}(\mathbf{u})}^{(n)}\right)$ and

$$
\left\|\mathbf{1}_{\mathbf{x}}^{(n, \text { out })} \mathbf{G}_{\mathbf{C}_{L}^{(n)}(\mathbf{x})}^{(n)}(E) \mathbf{1}_{\mathbf{x}}^{(n, i n t)}\right\| \leq \mathrm{e}^{-\gamma(m, L, n) L},
$$

where

$$
\gamma(m, L, n)=m\left(1+L^{-1 / 8}\right)^{N-n+1} .
$$

Otherwise, it is called $(E, m)$-singular $((E, m)-S)$.

Let us introduce the following:

Definition 4. Let $n \geq 1, E \in \mathbb{R}$ and $\alpha=3 / 2$.

(A) A cube $\mathbf{C}_{L}^{(n)}(\mathbf{v}) \subset \mathbb{R}^{n d}$ is called $E$-resonant $(E-R)$ if

$$
\operatorname{dist}\left[E, \sigma\left(\mathbf{H}_{\mathbf{C}_{L}^{(n)}(\mathbf{v})}^{(n)}\right)\right] \leq \mathrm{e}^{-L^{1 / 2}},
$$

Otherwise, it is called $E$-nonresonant $(E-R)$.

(B) A cube $\mathbf{C}_{L}^{(n)}(\mathbf{v}) \subset \mathbb{R}^{n d}$ is called $E$-completely nonresonant ( $E$-CNR), if it does not contain any $E-R$ cube of size $\geq L^{1 / \alpha}$. In particular $\mathbf{C}_{L}^{(n)}(\mathbf{v})$ is itself $E-\mathrm{NR}$.

We will also make use of the following notion,

Definition 5. A cube $\mathbf{C}_{L}^{(n)}(\mathbf{x})$ is $\mathcal{J}$-separable from $\mathbf{C}_{L}^{(n)}(\mathbf{y})$ if there exists a nonempty subset $\mathcal{J} \subset\{1, \ldots, n\}$ such that

$$
\left(\bigcup_{j \in \mathcal{J}} C_{L}^{(1)}\left(x_{j}\right)\right) \cap\left(\bigcup_{j \notin \mathcal{J}} C_{L}^{(1)}\left(x_{j}\right) \cup \bigcup_{j=1}^{n} C_{L}^{(1)}\left(y_{j}\right)\right)=\varnothing \text {. }
$$

A pair $\left(\mathbf{C}_{L}^{(n)}(\mathbf{x}), \mathbf{C}_{L}^{(n)}(\mathbf{y})\right)$ is separable if $|\mathbf{x}-\mathbf{y}| \geq 7 N L$ and if one of the cube is $\mathcal{J}$-separable from the other.
Lemma 6. Let $L \geq 1$.

(A) For any $\mathbf{x} \in \mathbb{Z}^{\text {nd }}$, there exists a collection of $n$-particle cubes $\mathbf{C}_{2 n L}^{(n)}\left(\mathbf{x}^{(\ell)}\right)$ with $\ell=1, \ldots, \kappa(n), \kappa(n)=n^{n}$, $\mathbf{x}^{(\ell)} \in \mathbb{Z}^{\text {nd }}$ such that if $\mathbf{y} \in \mathbb{Z}^{\text {nd }}$ satisfies $|\mathbf{y}-\mathbf{x}| \geq 7 N L$ and

$$
\mathbf{y} \notin \bigcup_{\ell=1}^{\kappa(n)} \mathbf{C}_{2 n L}^{(n)}\left(\mathbf{x}^{(\ell)}\right)
$$

then the cubes $\mathbf{C}_{L}^{(n)}(\mathbf{x})$ and $\mathbf{C}_{L}^{(n)}(\mathbf{y})$ are separable.

(B) Let $\mathbf{C}_{L}^{(n)}(\mathbf{y}) \subset \mathbb{R}^{n d}$ be an n-particle cube. Any cube $\mathbf{C}_{L}^{(n)}(\mathbf{x})$ with

$$
|\mathbf{y}-\mathbf{x}| \geq \max _{1 \leq i, j \leq n}\left|y_{i}-y_{j}\right|+5 N L
$$

is $\mathcal{J}$-separable from $\mathbf{C}_{L}^{(n)}(\mathbf{y})$ for some $\mathcal{J} \subset\{1, \ldots, n\}$.

Proof. See Appendix A.

3.2. The Multiparticle Wegner Estimates. We state below the Wegner estimates directly in a form suitable for our multiparticle multiscale analysis using assumption $(\mathbf{P})$.

Theorem 7. Assume that the random potential satisfies assumption $(\mathbf{P})$, then

(A) For any $E \in \mathbb{R}$

$$
\mathbb{P}\left\{\mathbf{C}_{L}^{(n)}(\mathbf{x}) \text { is not } E-C N R\right\} \leq L^{-p 4^{N-n}}
$$

(B)

$\mathbb{P}\left\{\exists E \in \mathbb{R}\right.$ neither $\mathbf{C}_{L}^{(n)}(\mathbf{x})$ nor $\mathbf{C}_{L}^{(n)}(\mathbf{y})$ is $\left.E-C N R\right\} \leq L^{-p 4^{N-n}}$,

where $p \geq 6 N d$, depends only on the fixed number of particles $N$ and the configuration dimension $d$.

Proof. See the articles $[15,16]$.

We also give the Combes-Thomas estimates in

Theorem 8. Let $H=-\Delta+W$ be a Schrödinger operator on $L^{2}\left(\mathbb{R}^{D}\right), E \in \mathbb{R}$ and $E_{0}=\inf \sigma(H)$. Set $\eta=\operatorname{dist}(E, \sigma(H))$. If $E$ is less than $E_{0}$, then for any $\gamma \in(0,1)$, we have that

$$
\left\|\mathbf{1}_{\mathbf{x}}(H-E)^{-1} \mathbf{1}_{\mathbf{y}}\right\| \leq \frac{1}{\left(1-\gamma^{2}\right) \eta} \mathrm{e}^{\gamma \sqrt{\eta d}} \mathrm{e}^{-\gamma \sqrt{\eta}|\mathbf{x}-\mathbf{y}|},
$$

for all $\mathbf{x}, \mathbf{y} \in \mathbb{R}^{D}$.

Proof. See the proof of Theorem 1 in [17].

We define the mass $m$ depending on the parameters $N, \gamma$, and the initial length scale $L$ in the following way:

$$
m:=\frac{2^{-N} \gamma L^{-1 / 4}}{3 \sqrt{2}}
$$


We recall below the geometric resolvent and the eigenfunction decay inequalities.

Theorem 9 (geometric resolvent inequality (GRI)). For $a$ given bounded $I_{0} \subset \mathbb{R}$. There is a positive constant $C_{\text {geom }}$ such that for $\mathbf{C}_{\ell}^{(n)}(\mathbf{x}) \subset \mathbf{C}_{L}^{(n)}(\mathbf{u}), \mathbf{A} \subset \mathbf{C}_{\ell}^{(n, i n t)}(\mathbf{x}), \mathbf{B} \subset \mathbf{C}_{L}^{(n)}(\mathbf{u}) \backslash \mathbf{C}_{\ell}^{(n)}(\mathbf{x})$ and $E \in I_{0}$, the following inequality holds true:

$$
\begin{gathered}
\left\|\mathbf{1}_{\mathbf{B}} \mathbf{G}_{\mathbf{C}_{L}^{(n)}(\mathbf{u})}^{(n)}(E) \mathbf{1}_{\mathbf{A}}\right\| \leq C_{\text {geom }} \cdot\left\|\mathbf{1}_{\mathbf{B}} \mathbf{G}_{\mathbf{C}_{L}^{(n)}(\mathbf{u})}^{(n)}(E) \mathbf{1}_{\mathbf{C}_{e}^{(n, i n t)}(\mathbf{x})}\right\| \cdot \\
\left\|\mathbf{1}_{\mathbf{C}_{e}^{(n, o u t)}(\mathbf{x})}\right\| \cdot\left\|\mathbf{1}_{\mathbf{C}_{e}^{(n, o u t)}(\mathbf{x})} \mathbf{G}_{\mathbf{C}_{e}^{(n)}(\mathbf{x})}^{(n)}(E) \mathbf{1}_{\mathbf{A}}\right\| .
\end{gathered}
$$

Proof. See [12], Lemma 2.5.4.

Theorem 10 (eigenfunctions decay inequality (EDI)). For every $E \in \mathbb{R}, \mathbf{C}_{\ell}^{(n)}(\mathbf{x}) \subset \mathbb{R}^{\text {nd }}$ and every polynomially bounded function $\Psi \in L^{2}\left(\mathbb{R}^{\text {nd }}\right)$ :

$\left\|\mathbf{1}_{\mathbf{C}_{1}^{(n)}(\mathbf{x})} \cdot \Psi\right\| \leq C \cdot\left\|\mathbf{1}_{\mathbf{C}_{\ell}^{(n, o u t)}(\mathbf{x})} \mathbf{G}_{\mathrm{C}_{\ell}^{(n)}(\mathbf{x})}^{(n)}(E) \mathbf{1}_{\mathrm{C}_{\ell}^{(\text {hint })}(\mathbf{x})}\right\| \cdot\left\|1_{\mathrm{C}_{\ell}^{(h, o u t)}(\mathbf{x})} \cdot \Psi\right\|$.

Proof. See Section 2.5 and Proposition 3.3.1. in [12].

\section{The Initial Bounds of the Multiparticle Multiscale Analysis}

In this Section, we denote by $E_{0}^{n}(\omega)$ the bottom of the spectrum of the Hamiltonian $\mathbf{H}_{\mathbf{C}_{L}^{(n)}(\mathbf{u})}^{(n)}(\omega)$ i.e., $E_{0}^{(n)}:=\inf \sigma\left(\mathbf{H}_{\mathbf{C}_{L}^{(n)}(\mathbf{u})}^{(n)}(\omega)\right)$. We give the following bound from the single-particle localization theory.

Theorem 11. Under the hypotheses (I) and (P), for any positive $p$, there exists a positive $L^{*}$ such that

$$
\mathbb{P}\left\{E_{0}^{(1)}(\omega) \leq L^{-1 / 2}\right\} \leq L^{-2 p 4^{N-1}}
$$

for all $L \geq L^{*}$.

Proof. See the book by Peter Stollmann [12].

Now, in the following statement, we show that the same result holds true for the multiparticle random Hamiltonian.

Theorem 12. Under the hypotheses (I) and (P), for any positive $p$ there exists a positive $L_{1}^{*}$ such that

$$
\mathbb{P}\left\{E_{0}^{(n)}(\omega) \leq L^{-1 / 2}\right\} \leq L^{-2 p 4^{N-n}}
$$

for all $L \geq L_{1}^{*}$.

Proof. We denote by $\mathbf{H}_{0}^{(n)}(\omega)$ the multiparticle random Hamiltonian without interaction. Observe that, since the interaction potential $\mathbf{U}$ is nonnegative we have

$$
E_{0}\left(\mathbf{H}_{\mathbf{C}_{L}^{(n)}(\mathbf{u})}^{(n)}(\omega)\right) \geq E_{0}^{(n)}(\omega)
$$

where $E_{0}^{(n)}(\omega)=\lambda_{1}^{(1)}(\omega)+\cdots+\lambda_{n}^{(1)}$ and the $\lambda_{i}^{(1)}$ are the eigenvalues of the single-particle random Hamiltonians $H_{C_{L}^{(1)}\left(u_{i}\right)}^{(1)}(\omega)$ $i=1, \ldots, n$. So, if $E_{0}^{(n)} \leq L^{-1 / 2}$, then for example $\lambda_{1}^{(1)} \leq L^{-1 / 2}$ and this implies the required probability bound of the assertion. $\square$

We are now ready to prove our initial length scale estimate of the multiparticle multiscale analysis given below.

Recall that the positive parameter $m$ is defined by $m=2^{-N} \gamma L^{-1 / 4} / 3 \sqrt{2}$.

Theorem 13. Assume that the hypotheses (I) and (P) hold true. Then there exists a positive $E^{*}$ such that

$$
\mathbb{P}\left\{\exists E \in\left(-\infty: E^{*}\right] \mathbf{C}_{L}^{(n)}(\mathbf{u}) \text { is }(E, m)-S\right\} \leq L^{-2 p 4^{N-n}},
$$

for $L \geq 0$ large enough.

Proof. Set $E^{*}:=(1 / 2) L^{-1 / 2}$. If the first eigenvalue $E_{0}^{(n)}(\omega)$ satisfies $E_{0}^{(n)}(\omega) \geq L^{-1 / 2}$, then for all energy $E \leq E^{*}$, we have:

$$
\begin{aligned}
\operatorname{dist}\left(E, \sigma\left(\mathbf{H}_{\mathbf{C}_{L}^{(n)}(\mathbf{u})}^{(n)}(\omega)\right)\right) & =E_{0}^{(n)}(\omega)-E \\
& \geq L^{-1 / 2}-\frac{1}{2} L^{-1 / 2} \\
& \geq \frac{1}{2} L^{-1 / 2} .
\end{aligned}
$$

Thus using the Combes-Thomas estimate Theorem 3.2

$$
\begin{aligned}
\| \mathbf{1}_{\mathbf{x}} \mathbf{G}_{\mathrm{C}_{L}^{(n)}(\mathbf{u})}^{(n)} & (E) \mathbf{1}_{\mathbf{y}} \| \leq 2 L^{1 / 2} \mathrm{e}^{\gamma \sqrt{\eta \bar{d}}|\mathbf{x}-\mathbf{y}|} \\
& \leq 2 L^{1 / 2} \mathrm{e}^{-\left(\gamma L^{-1 / 4}\right) / \sqrt{2}((L / 3)-\sqrt{d})} .
\end{aligned}
$$

Thus for $L \geq 0$ large enough depending on the dimension $d$, we get

$$
\begin{aligned}
& \left\|\mathbf{1}_{\mathbf{C}_{L}^{(\text {nout })}(\mathbf{u})} \mathbf{G}_{\mathbf{C}_{L}^{(n)}(\mathbf{u})}^{(n)}(E) \mathbf{1}_{\mathbf{C}_{L}^{(\text {mint })}(\mathbf{u})}\right\| \\
& \leq \sum_{\mathbf{x} \in \mathbf{C}_{L}^{(n, o u t)}(\mathbf{u}) \cap \mathbb{Z}^{\text {nd }}} 2 L^{1 / 2} \mathrm{e}^{-\left(\gamma L^{-1 / 4}\right) / \sqrt{2}((L / 3)-\sqrt{d})} \\
& \mathbf{y} \in \mathbf{C}_{L}^{(n, \text { int })}(\mathbf{u}) \cap \mathbb{Z}^{\text {nd }} \\
& \leq(2 L)^{2 n d} 2 L^{1 / 2} \mathrm{e}^{2^{N} m L} \text {. }
\end{aligned}
$$

Now, since $\gamma(m, L, n)=m\left(1+L^{-1 / 8}\right)^{N-n} \leq 2^{N} m$, for $L \geq 0$ large enough, we have that

$$
\left\|\mathbf{1}_{\mathbf{C}^{(n, o u t)}(\mathbf{u})} \mathbf{G}_{\mathbf{C}_{L}^{(n)}(\mathbf{u})}^{(n)}(E) \mathbf{1}_{\mathbf{C}_{L}^{(n, i n t)}(\mathbf{u})}\right\| \leq \mathrm{e}^{-\gamma(m, L, n) L} .
$$

The above analysis then implies that

$$
\begin{gathered}
\mathbb{P}\left\{\exists E \leq E^{*}: \mathbf{C}_{L}^{(n)}(\mathbf{u}) \text { is }(E, m)-\mathrm{S}\right\} \\
\mathbb{P}\left\{E_{0}^{(n)}(\omega) \leq L^{-1 / 2}\right\} \leq L^{-2 p 4^{N-n}} .
\end{gathered}
$$

Yielding the required result.

Below, we develop the induction step of the multiscale analysis and although the text overlaps with the paper [14], for the reader convenience we also give the detailed of the proofs of some important results.

\section{Multiscale Induction}

In the rest of the paper, we assume that $n \geq 2$ and $I_{0}$ is the interval from the previous Section. 
Recall the following facts from [2]: Consider a cube $\mathbf{C}_{L}^{(n)}(\mathbf{u})$ with $\mathbf{u}=\left(u_{1}, \ldots, u_{n}\right) \in\left(\mathbb{Z}^{d}\right)^{n}$. We define

$$
\Pi \mathbf{u}=\left\{u_{1}, \ldots, u_{n}\right\}
$$

and

$$
\Pi \mathbf{C}_{L}^{(n)}(\mathbf{u})=C_{L}^{(1)}\left(u_{1}\right) \cup \cdots \cup C_{L}^{(1)}\left(u_{n}\right) .
$$

Definition 14. Let $L_{0} \geq 3$ be a constant and $\alpha=3 / 2$. We define the sequence $\left\{L_{k}: k \geq 1\right\}$ recursively as follows

$$
L_{k}=\left\lfloor L_{k-1}^{\alpha}\right\rfloor, \quad \text { for all } k \geq 1 .
$$

Let $m$ be a positive constant. We also introduce the following property, namely the multiscale analysis bounds at any scale length $L_{k}$ and for any pair of separable cubes $\mathbf{C}_{L_{k}}^{(n)}(\mathbf{u})$ and $\mathbf{C}_{L_{k}}^{(n)}(\mathbf{v})$.

(DS. $k, n, N)$.

$$
\mathbb{P}\left\{\exists E \in I_{0}: \mathbf{C}_{L_{k}}^{(n)}(\mathbf{u}) \text { and } \mathbf{C}_{L_{k}}^{(n)}(\mathbf{v}) \text { are }(E, m)-S\right\} \leq L_{k}^{-2 p 4^{N-n}} \text {, }
$$

where $p \geq 6 \mathrm{Nd}$.

In both the single-particle and the multiparticle systems, given the results on the multiscale analysis property (DS.k, n, $\mathrm{N})$ above, one can deduce the localization results see for example the papers $[13,18]$ for those concerning the single-particle case and $[2,7]$ for multiparticle systems. We have the following

Definition 15 (fully/partially interactive). An $n$-particle cube $\mathrm{C}^{(n)} \subset \mathbb{Z}^{\text {nd }}$ is called fully interactive (FI) if

$$
\operatorname{diam} \Pi \mathbf{u}:=\max _{i \neq j}\left|u_{i}-u_{j}\right| \leq n\left(2 L_{0}+r_{0}\right),
$$

and partially interactive (PI) otherwise. cubes.

The following simple statement clarifies the notion of PI

Lemma 16. If a cube $\mathbf{C}_{L}^{(n)}(\mathbf{u})$ is PI, then there exists a subset $\mathcal{J} \subset\{1, \ldots, n\}$ with $1 \leq \operatorname{card} \mathcal{J} \leq n-1$ such that

$$
\operatorname{dist}\left(\Pi_{\mathcal{J}} \mathbf{C}_{L}^{(n)}(\mathbf{u}), \Pi_{\mathcal{J}} \mathbf{C}_{L}^{(n)}(\mathbf{u})\right) \geq r_{0},
$$

Proof. See Appendix B. it as

If $\mathbf{C}_{L}^{(n)}(\mathbf{u})$ is a PI cube by the above Lemma, we can write

$$
\mathbf{C}_{L}^{(n)}(\mathbf{u})=\mathbf{C}_{L}^{\left(n^{\prime}\right)}\left(\mathbf{u}^{\prime}\right) \times \mathbf{C}_{L}^{\left(n^{\prime \prime}\right)}\left(\mathbf{u}^{\prime \prime}\right)
$$

with,

$$
\operatorname{dist}\left(\Pi \mathbf{C}_{L}^{\left(n^{\prime}\right)}\left(\mathbf{u}^{\prime}\right), \Pi \mathbf{C}_{L}^{\left(n^{\prime \prime}\right)}\left(\mathbf{u}^{\prime \prime}\right)\right) \geq r_{0},
$$

where $\mathbf{u}^{\prime}=\mathbf{u}_{\mathcal{J}}=\left(u_{j}, j \in \mathcal{J}\right), \quad \mathbf{u}^{\prime \prime}=\left(u_{j} ; j \in \mathcal{J}\right) n^{\prime}=\operatorname{card} \mathcal{J}$ and $n^{\prime \prime}=$ card $\mathcal{F}$. Throughout, when we write a PI cube $\mathbf{C}_{L}^{(n)}(\mathbf{u})$ in the form (5.7) we implicitly assume that the projections satisfy (5.8). Let $\mathbf{C}_{L_{k}}^{\left(n^{\prime}\right)}\left(\mathbf{u}^{\prime}\right) \times \mathbf{C}_{L_{k}}^{\left(n^{\prime \prime}\right)}\left(\mathbf{u}^{\prime \prime}\right)$ be the decomposition of the PI cube $\mathbf{C}_{L_{k}}^{(n)}(\mathbf{u})$ and $\left\{\lambda_{i}, \varphi_{i}\right\}$ and $\left\{\mu_{j}, \phi_{j}\right\}$ be the eigen-

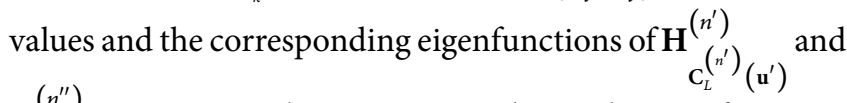
$\mathbf{H}_{\mathbf{C}_{L}^{\left(n^{\prime \prime}\right)}\left(\mathbf{u}^{\prime \prime}\right)}^{\left(n^{\prime \prime}\right)}$ respectively. Next, we can choose the eigenfunctions $\Psi_{i j}$ of $\mathbf{H}_{\mathrm{C}_{L}^{(n)}(\mathbf{u})}^{(n)}(\omega)$ as tensor products:

$$
\Psi_{i j}=\varphi_{i} \otimes \phi_{j} .
$$

The eigenfunctions appearing in subsequent arguments and calculation will be assumed normalized. Now, we turn to geometric properties of FI cubes.

Lemma 17. Let $n \geq 1, L \geq 2 r_{0}$ and consider two FI cubes $\mathbf{C}_{L}^{(n)}(\mathbf{x})$ and $\mathbf{C}_{L}^{(n)}(\mathbf{y})$ with $|\mathbf{x}-\mathbf{y}| \geq 7 n L$. Then

$$
\Pi \mathbf{C}_{L}^{(n)}(\mathbf{x}) \cap \Pi \mathbf{C}_{L}^{(n)}(\mathbf{y})=\varnothing .
$$

Proof. See Appendix C.

Given an $n$-particle cube $\mathbf{C}_{L}^{(n)}(\mathbf{u})$ and $E \in \mathbb{R}$, we denote by

(i) $\quad M_{\mathrm{PI}}^{\text {sep }}\left(\mathbf{C}_{L_{k+1}}^{(n)}(\mathbf{u}), E\right)$ the maximal number of pairwise separable $(E, m)$-singular PI cubes $\mathbf{C}_{L_{k}}^{(n)}\left(\mathbf{u}^{(j)}\right) \subset \mathbf{C}_{L_{k+1}}^{(n)}(\mathbf{u})$;

(ii) by $M_{\mathrm{PI}}\left(\mathbf{C}_{L_{k+1}}^{(n)}(\mathbf{u}), E\right)$, the maximal number of (not necessary separable) $(E, m)$-singular PI-cubes $\mathbf{C}_{L_{k}}^{(n)}\left(\mathbf{u}^{(j)}\right)$ contain in $\mathbf{C}_{L_{k+1}}^{(n)}(\mathbf{u})$ with $\mathbf{u}^{(j)}, \mathbf{u}^{\left(j^{\prime}\right)} \mathbb{Z}^{\text {nd }}$ and $\left|\mathbf{u}^{(j)}-\mathbf{u}^{\left(j^{\prime}\right)}\right| \geq 7 N L_{k}$ for all $j \neq j^{\prime}$;

(iii) $M_{\mathrm{FI}}\left(\mathbf{C}_{L_{k+1}}^{(n)}(\mathbf{u}), E\right)$ the maximal number of $(E, m)$ -singular FI cubes $\mathbf{C}_{L_{k}}^{(n)}\left(\mathbf{u}^{(j)}\right) \subset \mathbf{C}_{L_{k+1}}^{(n)}(\mathbf{u})$ with $\left|\mathbf{u}^{(j)}-\mathbf{u}^{\left(j^{\prime}\right)}\right| \geq 7 N L_{k}$ for all $j \neq j^{\prime}$ (Note that by Lemma 17; two FI cubes $\mathbf{C}_{L_{k}}^{(n)}\left(\mathbf{u}^{(j)}\right)$ and $\mathbf{C}_{L_{k}}^{(n)}\left(\mathbf{u}^{\left(j^{\prime}\right)}\right)$ with $\left|\mathbf{u}^{(j)}-\mathbf{u}^{\left(j^{\prime}\right)}\right| \geq 7 N L_{k}$ are automatically separable);

(iv) $M_{\mathrm{PI}}\left(\mathbf{C}_{L_{k+1}}^{(n)}(\mathbf{u}), I\right):=\sup _{E \in I} M_{\mathrm{PI}}\left(\mathbf{C}_{L_{k+1}}^{(n)}(\mathbf{u}), E\right)$;

(v) $M_{\mathrm{FI}}\left(\mathbf{C}_{L_{k+1}}^{(n)}(\mathbf{u}), I\right):=\sup _{E \in I} M_{\mathrm{FI}}\left(\mathbf{C}_{L_{k+1}}^{(n)}(\mathbf{u}), E\right)$;

(vi) $M\left(\mathbf{C}_{L_{k+1}}^{(n)}(\mathbf{u}), E\right)$ the maximal number of $(E, m)$ -singular cubes $\mathbf{C}_{L_{k}}^{(n)}\left(\mathbf{u}^{(j)}\right) \subset \mathbf{C}_{L_{k+1}}^{(n)}(\mathbf{u}) \quad$ with $\operatorname{dist}\left(\mathbf{u}^{(j)}, \partial \mathbf{C}_{L_{k+1}}^{(n)}(\mathbf{u})\right) \geq 2 L_{k}$ and $\left|\mathbf{u}^{(j)}-\mathbf{u}^{\left(j^{\prime}\right)}\right| \geq 7 N L_{k}$ for all $j \neq j^{\prime}$;

(vii) $M^{\text {sep }}\left(\mathbf{C}_{L_{k+1}}^{(n)}(\mathbf{u}), E\right)$ the maximal number of pairwise separable $(E, m)$-singular cube $\mathbf{C}_{L_{k}}^{(n)}\left(\mathbf{u}^{(j)}\right) \subset \mathbf{C}_{L_{k+1}}^{(n)}(\mathbf{u})$;

Clearly,

$$
M_{\mathrm{PI}}\left(\mathbf{C}_{L_{k+1}}^{(n)}(\mathbf{u}), E\right)+M_{\mathrm{FI}}\left(\mathbf{C}_{L_{k+1}}^{(n)}(\mathbf{u}), E\right) \geq M\left(\mathbf{C}_{L_{k+1}}^{(n)}(\mathbf{u}), E\right) .
$$

5.1. Pairs of Partially Interactive Cubes. Let $\mathbf{C}_{L_{k+1}}^{(n)}(\mathbf{u})=\mathbf{C}_{L_{k+1}}^{\left(n^{\prime}\right)}\left(\mathbf{u}^{\prime}\right) \times \mathbf{C}_{L_{k+1}}^{\left(n^{\prime \prime}\right)}\left(\mathbf{u}^{\prime \prime}\right)$ be a PI-cube. We also write $\mathbf{x}=\left(\mathbf{x}^{\prime}, \mathbf{x}^{\prime \prime}\right)$ for any point $\mathbf{x} \in \mathbf{C}_{L_{k+1}}^{(n)}(\mathbf{u})$, in the same way as 

$\left(\mathbf{u}^{\prime}, \mathbf{u}^{\prime \prime}\right)$. So the corresponding Hamiltonian $\mathbf{H}_{\mathbf{C}_{L_{k+1}}^{(n)}(\mathbf{n})}^{(n)}$ is written
in the form:

$$
\begin{aligned}
\mathbf{H}_{\mathrm{C}_{L_{k+1}}^{(n)}(\mathbf{u})}^{(n)} \boldsymbol{\Psi}(\mathbf{x})= & (-\Delta \Psi)(\mathbf{x})+\left[\mathbf{U}\left(\mathbf{x}^{\prime}\right)+\mathbf{V}\left(\mathbf{x}^{\prime}, \omega\right)\right. \\
& \left.+\mathbf{U}\left(\mathbf{x}^{\prime \prime}\right)+\mathbf{V}\left(\mathbf{x}^{\prime \prime}, \omega\right)\right] \Psi(\mathbf{x})
\end{aligned}
$$

or in compact form:

$$
\mathbf{H}_{\mathbf{C}_{L_{k+1}}^{(n)}(\mathbf{u})}^{(n)}=\underset{\mathbf{C}_{L_{k+1}}^{\left(n^{\prime}\right)}\left(\mathbf{u}^{\prime}\right)}{\left(n^{\prime}\right)} \otimes \mathbf{I}+\mathbf{I} \otimes \mathbf{H}_{\mathbf{C}_{L_{k+1}}^{\left(n^{\prime \prime}\right)}\left(\mathbf{u}^{\prime \prime}\right)}^{\left(n^{\prime \prime}\right)} .
$$

We denote by $\mathbf{G}^{\left(n^{\prime}\right)}\left(\mathbf{u}^{\prime}, \mathbf{v}^{\prime} ; E\right)$ and $\mathbf{G}^{\left(n^{\prime \prime}\right)}\left(\mathbf{u}^{\prime \prime}, \mathbf{v}^{\prime \prime} ; E\right)$ the corresponding Green functions respectively. Introduce the following notions:

Definition 18 (see [10]). Let $1 \leq n \leq N$ and $E \in \mathbb{R}$. Consider a PI cube $\mathbf{C}^{(n)}=\mathbf{C}_{L}^{\left(n^{\prime}\right)}\left(\mathbf{u}^{\prime}\right) \times \mathbf{C}_{L}^{\left(n^{\prime \prime}\right)}\left(\mathbf{u}^{\prime \prime}\right)$. Then $\mathbf{C}_{L}^{(n)}(\mathbf{u})$ is called E-highly nonresonant (E-HNR) if

(i) for all $\mu_{j} \in \sigma\left(\begin{array}{c}\mathbf{H}_{\left(n^{\prime \prime}\right)}^{\left(n^{\prime \prime}\right)}\left(\mathbf{u}^{\prime \prime}\right) \\ \mathbf{C}_{L}\end{array}\right)$, the cube $\mathbf{C}_{L}^{\left(n^{\prime}\right)}\left(\mathbf{u}^{\prime}\right)$ is $\left(E-\mu_{j}\right)$-CNR.

(ii) for all $\lambda_{i} \in \sigma\left(\begin{array}{c}\mathbf{H}_{\mathbf{C}_{L}^{\left(n^{\prime}\right)}\left(\mathbf{u}^{\prime}\right)}^{\left(n^{\prime}\right)} \\ \mathbf{C}^{\prime}\end{array}\right)$, the cube $\mathbf{C}_{L}^{\left(n^{\prime \prime}\right)}\left(\mathbf{u}^{\prime \prime}\right)$ is $\left(E-\lambda_{i}\right)$-CNR.

Definition 19 ((E,m)-tunnelling). Let $1 \leq n \leq N, E \in \mathbb{R}$ and a positivem. ConsideraPIcube $\mathbf{C}_{L}^{(n)}(\mathbf{u})=\mathbf{C}_{L}^{\left(n^{\prime}\right)}\left(\mathbf{u}^{\prime}\right) \times \mathbf{C}_{L}^{\left(n^{\prime \prime}\right)}\left(\mathbf{u}^{\prime \prime}\right)$. Then $\mathbf{C}_{L}^{(n)}(\mathbf{u})$ is called

(i) $(E, m) \quad$ left-tunnelling $\quad((E, m)$-LT $) \quad$ if $\exists \mu_{j} \in \sigma\left(\begin{array}{c}\mathbf{H}_{\left(n^{\prime \prime}\right)}^{\left(n^{\prime \prime}\right)}\left(\mathbf{u}^{\prime \prime}\right) \\ \mathbf{C}_{L}\end{array}\right)$ such that $\mathbf{C}_{L}^{\left(n^{\prime}\right)}\left(\mathbf{u}^{\prime}\right)$ contains two separable $\left(E-\mu_{j}\right)$-S cubes $\mathbf{C}_{\ell}^{\left(n^{\prime}\right)}\left(\mathbf{v}_{1}\right)$ and $\mathbf{C}_{\ell}^{\left(n^{\prime}\right)}\left(\mathbf{v}_{2}\right)$ with $L=\left\lfloor\ell^{\alpha}\right\rfloor+1$. Otherwise it is called $(E, m)$ nonleft-tunnelling $((E, m)$-NLT).

(ii) $(E, m)$ right-tunnelling $((E, m)-\mathrm{RT}) \quad$ if $\exists \lambda_{i} \in \sigma\left(\begin{array}{c}\mathbf{H}_{\left(n^{\prime}\right)}^{\left(n^{\prime}\right)}\left(\mathbf{u}^{\prime}\right) \\ \mathbf{C}_{L}\end{array}\right)$ such that $\mathbf{C}_{L}^{\left(n^{\prime \prime}\right)}\left(\mathbf{u}^{\prime \prime}\right)$ contains two separable $\left(E-\lambda_{i}\right)-S$ cubes $\mathbf{C}_{\ell}^{\left(n^{\prime \prime}\right)}\left(\mathbf{v}_{1}\right)$ and $\mathbf{C}_{\ell}^{\left(n^{\prime \prime}\right)}\left(\mathbf{v}_{2}\right)$ with $L=\left\lfloor\ell^{\alpha}\right\rfloor+1$. Otherwise it is called $(E, m)$ nonright-tunnelling $((E, m)$-NRT).

(iii) $(E, m)$-tunnelling $((E, m)$-T) if either it is $(E, m)$-LT or $(E, m)$-RT. Otherwise it is called $(E, m)$-nontunnelling $((E, m)-\mathrm{NT})$.

We reformulate and prove Lemma 3.18 from [10] in our context.
Lemma 20. Let $E \in \mathbb{R}$. If a PI cube $\mathbf{C}_{L}^{(n)}(\mathbf{u})=\mathbf{C}_{L}^{\left(n^{\prime}\right)}\left(\mathbf{u}^{\prime}\right) \times$ $\mathbf{C}_{L}^{\left(n^{\prime \prime}\right)}\left(\mathbf{u}^{\prime \prime}\right)$ is not E-HNR, then

(i) either there exist $L^{1 / \alpha} \leq \ell \leq L, \quad \mathbf{x} \in \mathrm{C}_{L}^{\left(n^{\prime}\right)}\left(\mathbf{u}^{\prime}\right)$
such that the n-particle rectangle $\mathbf{C}^{(n)}=\mathbf{C}_{\ell}^{\left(n^{\prime}\right)}(\mathbf{x}) \times \mathbf{C}_{L}^{\left(n^{\prime \prime}\right)}\left(\mathbf{u}^{\prime \prime}\right) \subset \mathbf{C}_{L}^{(n)}(\mathbf{u})$ is $E-\mathbf{R}$,

(ii) or there exist $L^{1 / \alpha} \leq \ell \leq L, \quad \mathbf{x} \in \mathbf{C}_{L}^{\left(n^{\prime \prime}\right)}\left(\mathbf{u}^{\prime \prime}\right)$ such that the n-particle rectangle $\mathbf{C}^{(n)}=\mathbf{C}_{L}^{\left(n^{\prime}\right)}\left(\mathbf{u}^{\prime}\right) \times \mathbf{C}_{\ell}^{\left(n^{\prime \prime}\right)}(\mathbf{x}) \subset \mathbf{C}_{L}^{(n)}(\mathbf{u})$ is E-R.

Proof. By Definition 18, if $\mathbf{C}_{L}^{(n)}(\mathbf{u})$ is not E-HNR then either (a) there exists $\mu_{j} \in \sigma\left(\begin{array}{c}\mathbf{H}^{\left(n^{\prime \prime}\right)} \\ \mathbf{C}_{L}^{\left(n^{\prime \prime}\right)}\left(\mathbf{u}^{\prime \prime}\right)\end{array}\right)$ such that $\mathbf{C}_{L}^{\left(n^{\prime}\right)}\left(\mathbf{u}^{\prime}\right)$ is not $E-\mu_{j}$-CNR or (b) there exists $\lambda_{i} \in \sigma\left(\begin{array}{c}\mathbf{H}_{\left(n^{\prime}\right)}^{\left(n^{\prime}\right)} \\ \mathbf{C}_{L}^{\left(n^{\prime}\right)}\end{array}\right)$ such that $\mathrm{C}_{L}^{\left(n^{\prime \prime}\right)}\left(\mathbf{u}^{\prime \prime}\right)$ is not $E-\lambda_{i}$-CNR. Let us first focus on case (a). Since $\mathbf{C}_{L}^{\left(n^{\prime}\right)}\left(\mathbf{u}^{\prime}\right)$ is not $E-\mu_{j}$-CNR there exist $L^{1 / \alpha} \leq \ell \leq L$, $\mathbf{x} \in \mathbf{C}_{L}^{\left(n^{\prime}\right)}\left(\mathbf{u}^{\prime}\right)$ such that $\mathbf{C}_{\ell}^{\left(n^{\prime}\right)}(\mathbf{x}) \subset \mathbf{C}_{L}^{\left(n^{\prime}\right)}\left(u^{\prime}\right)$ and $\mathbf{C}_{\ell}^{\left(n^{\prime}\right)}(\mathbf{x})$ is

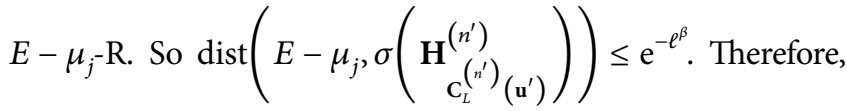
there exists $\eta \in \sigma\left(\begin{array}{c}\mathbf{H}_{\mathbf{C}_{\ell}^{\left(n^{\prime}\right)}(\mathbf{x})}^{\left(n^{\prime}\right)} \\ { }_{n^{\prime}}\end{array}\right)$ such that $\left|E-\mu_{j}-\eta\right| \leq \mathrm{e}^{-\ell^{\beta}}$. Now consider $\mathbf{C}^{(n)}=\mathbf{C}_{\ell}^{\left(n^{\prime}\right)}(\mathbf{x}) \times \mathbf{C}_{L}^{\left(n^{\prime \prime}\right)}\left(\mathbf{u}^{\prime \prime}\right)$, since the cube $\mathbf{C}_{L}^{(n)}(\mathbf{u})$ is

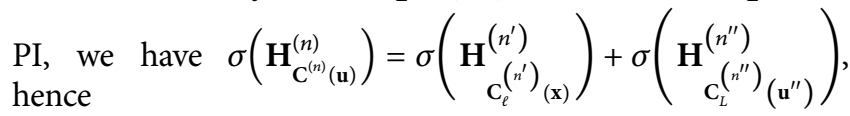

$$
\operatorname{dist}\left(E, \sigma\left(\mathbf{H}_{\mathbf{C}^{(n)}}^{(n)}\right)\right) \leq\left|E-\mu_{j}-\eta\right| \leq \mathrm{e}^{-\ell^{\beta}} .
$$

Thus $\mathrm{C}^{(n)}$ is $E$-R. The same arguments shows that case (ii) arises when (b) occurs.

Lemma 21. Let $E \in I$ and $\mathbf{C}_{L_{k}}^{(n)}(\mathbf{u})$ be a PI cube. Assume that $\mathbf{C}_{L_{k}}^{(n)}(\mathbf{u})$ is $(E, m)-N T$ and E-HNR. Then $\mathbf{C}_{L_{k}}^{(n)}(\mathbf{u})$ is $(E, m)-N S$.

Proof. Let $\mathbf{C}_{L_{k}}^{\left(n^{\prime}\right)}\left(\mathbf{u}^{\prime}\right) \times \mathbf{C}_{L_{k}}^{\left(n^{\prime \prime}\right)}\left(\mathbf{u}^{\prime \prime}\right)$ be the decomposition of the PI cube $\mathbf{C}_{L_{k}}^{(n)}(\mathbf{u})$. Let $\left\{\lambda_{i}, \varphi_{i}\right\}$ and $\left\{\mu_{j}, \phi_{j}\right\}$ be the eigenvalues and corresponding eigenfunctions of $\mathbf{H}^{\left(n^{\prime}\right)}{ }_{\mathrm{C}_{L_{k}}^{\left(n^{\prime}\right)}\left(\mathbf{u}^{\prime}\right)}$ and $\mathbf{H}^{\left(n^{\prime \prime}\right)} \mathbf{C}_{L_{k}}^{\left(n^{\prime \prime}\right)}\left(\mathbf{u}^{\prime \prime}\right)$ respectively. Then, we can choose the eigenvectors $\Psi_{i j}$ and corresponding eigenvalues $E_{i j}$ of $\mathbf{H}_{\mathbf{C}_{L_{L}}^{(n)}(\mathbf{u})}^{(n)}$ as follows

$$
\Psi_{i j}=\varphi_{i} \otimes \phi_{j}, \quad E_{i j}=\lambda_{i}+\mu_{j} .
$$

By the assumed E-HNR property of the cube $\mathbf{C}_{L_{k}}^{(n)}(\mathbf{u})$, for all eigenvalues $\lambda_{i}$ one has $\mathbf{C}_{L_{k}}^{\left(n^{\prime \prime}\right)}\left(\mathbf{u}^{\prime \prime}\right)$ is $E-\lambda_{i}$-CNR. Next, by assumption of $(E, m)-\mathrm{NT}, \mathbf{C}_{L_{k}}^{\left(n^{\prime \prime}\right)}\left(\mathbf{u}^{\prime \prime}\right)$ does not contain any pair of separable $\left(E-\lambda_{i}, m\right)$-S cubes of radius $L_{k-1}$ therefore by Lemma $26, M\left(\mathbf{C}_{L_{k+1}}^{(n)}(\mathbf{u}), E-\lambda_{i}\right) \leq n^{n}+2$ and the cube is also $\left(E-\lambda_{i}, m\right)$-NS, yielding 


$$
\max _{\lambda_{i}} \max _{\substack{\mathbf{v}^{\prime \prime} \in \partial^{-} \mathbf{C}_{L_{k}}^{\left(n^{\prime \prime}\right)}\left(\mathbf{u}^{\prime \prime}\right)\\}}\left|\mathbf{G}^{\left(n^{\prime \prime}\right)}\left(\mathbf{u}^{\prime \prime}, \mathbf{v}^{\prime \prime}, E-\lambda_{i}\right)\right| \leq \mathrm{e}^{-\gamma\left(m, L_{k}, n^{\prime \prime}\right) L_{k}} .
$$

The same analysis for $\mathbf{C}_{L_{k}}^{\left(n^{\prime}\right)}\left(\mathbf{u}^{\prime}\right)$ also gives

$$
\max _{\mu_{j}} \max _{\mathbf{v}^{\prime} \in \partial^{-} \mathbf{C}_{L_{k}}^{\left(n^{\prime}\right)}(\mathbf{u})}\left|\mathbf{G}_{\mathbf{C}_{L_{k}}^{\left(n^{\prime}\right)}\left(n^{\prime}\right)}^{\left(\mathbf{u}^{\prime}\right)}\left(\mathbf{u}^{\prime}, \mathbf{v}^{\prime}, E-\mu_{j}\right)\right| \leq \mathrm{e}^{-\gamma\left(m, L_{k}, n^{\prime}\right) L_{k}} .
$$

For any $\mathbf{v} \in \partial^{-} \mathbf{C}_{L_{k}}^{(n)}(\mathbf{u}),|\mathbf{u}-\mathbf{v}|=L_{k}$ thus either $\left|\mathbf{v}^{\prime}-\mathbf{u}^{\prime}\right|=L_{k}$ or $\left|\mathbf{v}^{\prime \prime}-\mathbf{u}^{\prime \prime}\right|=L_{k}$ consider first the latter case. Then we have

$$
\begin{aligned}
\left|\mathbf{G}^{(n)}(\mathbf{u}, \mathbf{v} ; E)\right|= & \left|\sum_{i j} \frac{\varphi_{i}\left(\mathbf{u}^{\prime}\right) \varphi_{i}\left(\mathbf{v}^{\prime}\right) \phi_{j}\left(\mathbf{u}^{\prime \prime}\right) \phi_{j}\left(\mathbf{v}^{\prime \prime}\right)}{E-\lambda_{i}-\mu_{j}}\right| \\
\leq & \sum_{i}\left|\varphi_{i}\left(\mathbf{u}^{\prime}\right) \varphi_{i}\left(\mathbf{v}^{\prime}\right)\right| \cdot\left|\mathbf{G}^{\left(n^{\prime \prime}\right)}\left(\mathbf{u}^{\prime \prime}, \mathbf{v}^{\prime \prime}, E-\lambda_{i}\right)\right| \\
\leq & \left(2 L_{k}+1\right)^{(n-1) d} \max _{\lambda_{i}} \max _{\mathbf{v}^{\prime \prime} \partial^{-} \mathbf{c}_{L_{k}}^{\prime \prime}\left(\mathbf{u}^{\prime \prime}\right)} \\
& \cdot\left|\mathbf{G}^{\left(n^{\prime \prime}\right)}\left(\mathbf{u}^{\prime \prime}, \mathbf{v}^{\prime \prime}, E-\lambda_{i}\right)\right|, \quad \text { since }\|\varphi\|_{\infty} \\
\leq & 1 \leq\left(2 L_{k}+1\right)^{(n-1) d} \cdot \mathrm{e}^{-\gamma\left(m, L_{k}, n-1\right) L_{k}} \\
= & \mathrm{e}^{-\left[\gamma\left(m, L_{k}, n-1\right)-L_{k}^{-1} \ln \left(2 L_{k}+1\right)^{(n-1) d}\right] L_{k}} .
\end{aligned}
$$

But by definition

$$
\gamma\left(m, L_{k}, n\right)=m\left(1+L_{k}^{-1 / 8}\right)^{N-n+1},
$$

For $2 \leq n \leq N$

$$
\gamma\left(m, L_{k}, n-1\right)-\gamma\left(m, L_{k}, n\right) \geq L_{k}^{-1} \ln \left(2 L_{k}+1\right)^{(n-1) d} .
$$

Indeed, setting $C_{1}=2^{-N} \gamma / 3 \sqrt{2}$,

$$
\begin{aligned}
\gamma\left(m, L_{k}, n-1\right) & -\gamma\left(m, L_{k}, n\right)=m L_{k}^{-1 / 8}\left(1+L_{k}^{-1 / 8}\right)^{N-n+1} \\
& \geq C_{1} L_{0}^{-1 / 2} L_{k}^{-1 / 8}\left(1+L_{k}^{-1 / 8}\right)^{N-n+1} \geq C_{1} L_{k}^{-5 / 8},
\end{aligned}
$$

and for $L_{0}$ sufficiently large, hence $L_{k}$

$L_{k}^{-1} \ln \left(2 L_{k}+1\right)^{(n-1) d} \leq L_{k}^{-1}(n-1) d\left(3 L_{k}\right)^{3 / 8} \leq C_{1} L_{k}^{-5 / 8}$.

Thus, $\mathbf{C}_{L_{k}}^{(n)}(\mathbf{u})$ is $(E, m)$-NS. Finally, the case $\left|\mathbf{u}^{\prime}-\mathbf{v}^{\prime}\right|=L_{k}$ is similar.

Lemma 22. Let $2 \leq n \leq N$ and assume property $\left(\right.$ DS. $\left.k, n^{\prime}, N\right)$ for any $n^{\prime} \in[1, n)$. Then for any PI cube $\mathbf{C}_{L_{k+1}}^{(n)}(\mathbf{y})$ one has

$$
\mathbb{P}\left\{\exists E \in I, \mathbf{C}_{L_{k+1}}^{(n)}(\mathbf{y}) \text { is }(E, m)-T\right\} \leq \frac{1}{2} L_{k+1}^{-4 p 4^{N-n}} .
$$

Proof. Consider a PI cube $\mathbf{C}_{L_{k+1}}^{(n)}(\mathbf{y})=\mathbf{C}_{L_{k+1}}^{\left(n^{\prime}\right)}\left(\mathbf{y}^{\prime}\right) \times \mathbf{C}_{L_{k+1}}^{\left(n^{\prime \prime}\right)}\left(\mathbf{y}^{\prime \prime}\right)$. By Definition 19, we have that the event

$$
\left\{\exists E \in I: \mathbf{C}_{L_{k+1}}^{(n)}(\mathbf{y}) \text { is }(E, m)-\mathrm{T}\right\} .
$$

is contained in the union

$$
\begin{aligned}
& \left\{\exists E \in I: \mathbf{C}_{L_{k+1}}^{(n)}(\mathbf{y}) \text { is }(E, m)-\mathrm{RT}\right\} \\
& \cup\left\{\exists E \in I: \mathbf{C}_{L_{k+1}}^{(n)}(\mathbf{y}) \text { is }(E, m)-\mathrm{LT}\right\} .
\end{aligned}
$$

Now, since $E \in I$ and $\mu_{j} \geq 0$ we have $E-\mu_{j} \leq E^{*}$. So for any $j$, $E-\mu_{j} \in I$. Further, using property $\left(\right.$ DS. $\left.k, n^{\prime}, N\right)$ we have

$$
\begin{gathered}
\mathbb{P}\left\{\exists E \in I: \mathbf{C}_{L_{k+1}}^{(n)}(\mathbf{y}) \text { is }(E, m)-\mathrm{RT}\right\} \\
\leq \frac{\left|\mathbf{C}_{L_{k+1}}^{\left(n^{\prime}\right)}\left(\mathbf{y}^{\prime}\right)\right|^{2}}{2}\left|\mathbf{C}_{L_{k+1}}^{\left(n^{\prime \prime}\right)}\left(\mathbf{y}^{\prime \prime}\right)\right| L_{k}^{-2 p 4^{N-n^{\prime}}} \\
\leq C(n, N, d) L_{k+1}^{-2 p 4^{(N-(n-1)} / \alpha+3(n-1) d} .
\end{gathered}
$$

A similar argument also shows that

$$
\begin{aligned}
\mathbb{P} & \left\{\exists E \in I: \mathrm{C}_{\mathrm{L}_{k+1}}^{(n)}(\mathbf{y}) \text { is }(E, m)-\mathrm{LT}\right\} \\
& \leq C(n, N, d) L_{k+1}^{-2 p 4^{(N-(n-1))} / \alpha+3(n-1) d}
\end{aligned}
$$

so that

$$
\begin{gathered}
\mathbb{P}\left\{\exists E \in I: \mathbf{C}_{\mathrm{L}_{k+1}}^{(n)}(\mathbf{y}) \text { is }(E, m)-\mathrm{LT}\right\} \\
\leq C(n, N, d) L_{k+1}^{-2 p 4^{(N-(n-1))} / \alpha+3(n-1) d},
\end{gathered}
$$

The assertion follows by observing that $2 p 4^{(N-(n-1))} / \alpha-3(n-1) d \geq 4 p 4^{N-n}$ for $\alpha=3 / 2$ provided $L_{0}$ is large enough and $p \geq 4 \alpha N d=6 \mathrm{Nd}$.

Theorem 23. Let $1 \leq n \leq N$. There exists $L_{1}^{*}=L_{1}^{*}(N, d) \geq 0$ such that if $L_{0} \geq L_{1}^{*}$ and if for $k \geq 0\left(D S . k, n^{\prime}, N\right)$ holds true for any $n^{\prime} \in[1, n)$ then $(\boldsymbol{D S} . k+1, n, N)$ holds true for any pair of separable PI cubes $\mathbf{C}_{L_{k+1}}^{(n)}(\mathbf{x})$ and $\mathbf{C}_{L_{k+1}}^{(n)}(\mathbf{y})$.

Proof. Let $\mathbf{C}_{L_{k+1}}^{(n)}(\mathbf{x})$ and $\mathbf{C}_{L_{k+1}}^{(n)}(\mathbf{y})$ be two separable PI cubes. Consider the events:

$$
\begin{aligned}
B_{k+1} & =\left\{\exists E \in I: \mathbf{C}_{L_{k+1}}^{(n)}(\mathbf{x}) \text { and } \mathbf{C}_{L_{k+1}}^{(n)}(\mathbf{y}) \text { are }(E, m)-\mathrm{S}\right\}, \\
R & =\left\{\exists E \in I: \text { neither } \mathbf{C}_{L_{k+1}}^{(n)}(\mathbf{x}) \text { nor } \mathbf{C}_{L_{k+1}}^{(n)}(\mathrm{y}) \text { is } E-\mathrm{HNR}\right\}, \\
T_{\mathbf{x}} & =\left\{\exists E \in I: \mathbf{C}_{L_{k+1}}^{(n)}(\mathbf{x}) \text { is }(E, m)-\mathrm{T}\right\}, \\
T_{\mathbf{y}} & =\left\{\exists E \in I: \mathbf{C}_{L_{k+1}}^{(n)}(\mathbf{y}) \text { is }(E, m)-\mathrm{T}\right\} .
\end{aligned}
$$

If $\omega \in B_{k+1} \backslash R$ then $\forall E \in I \mathbf{C}_{L_{k+1}}^{(n)}(\mathbf{x})$ or $\mathbf{C}_{L_{k+1}}^{(n)}(\mathbf{y})$ is $E$-HNR, then it must be $(E, m)$-T: otherwise it would have been $(E, m)$-NS by Lemma 21 . Similarly, if $\mathbf{C}_{L_{k+1}}^{(n)}(\mathbf{x})$ is $E$-HNR, then it must be $(E, m)$-T. This implies that

$$
B_{k+1} \subset R \cup T_{\mathbf{x}} \cup T_{\mathbf{y}} .
$$

Therefore,

$$
\begin{aligned}
\mathbb{P}\left\{B_{k+1}\right\} & \leq \mathbb{P}\{R\}+\mathbb{P}\left\{T_{\mathrm{x}}\right\}+\mathbb{P}\left\{T_{\mathrm{y}}\right\} \\
& \leq \mathbb{P}\{R\}+\frac{1}{2} L_{k+1}^{-4 p 4^{N-n}}+\frac{1}{2} L_{k+1}^{-4 p 4^{N-n}} .
\end{aligned}
$$

Next by combining Theorem 7 and Lemma 20 we obtain that $\mathbb{P}\{R\} \leq L_{k+1}^{-4^{N} p}$. Finally

$$
\mathbb{P}\left\{\mathrm{B}_{k+1}\right\} \leq \mathrm{L}_{k+1}^{-4^{N} p}+\mathrm{L}_{k+1}^{-4 p 4^{N-n}} \leq \mathrm{L}_{k+1}^{-2 p 4^{N-n}} .
$$


For subsequent calculations and proofs, we give the following two Lemmas.

Lemma 24. If $M\left(\mathbf{C}_{L_{k+1}}^{(n)}(\mathbf{u}), E\right) \geq \kappa(n)+2$ with $\kappa(n)=n^{n}$, then $M^{\text {sep }}\left(\mathbf{C}_{L_{k+1}}^{(n)}(\mathbf{u}), E\right) \geq 2$. Similarly if $M_{\mathrm{PI}}\left(\mathbf{C}_{L_{k+1}}^{(n)}(\mathbf{u}), E\right) \geq \kappa(n)+2$ then $M_{\mathrm{PI}}^{\mathrm{sep}}\left(\mathbf{C}_{L_{k+1}}^{(n)}(\mathbf{u}), E\right) \geq 2$.

Proof. See Appendix D.

Lemma 25. With the above notations, assume that $\left(D S . k-1, n^{\prime}, N\right)$ holds true for all $n^{\prime} \in[1, n)$ then

$\mathbb{P}\left\{M_{\mathrm{PI}}\left(\mathbf{C}_{L_{k+1}}^{(n)}(\mathbf{u}), I\right) \geq \kappa(n)+2\right\} \leq \frac{3^{2 n d}}{2} L_{k+1}^{2 n d}\left(L_{k}^{-4^{N} p}+L_{k}^{-4 p 4^{N-n}}\right)$.

Proof. See Appendix E.

5.2. Pairs of Fully Interactive Cubes. Our aim now is to prove $(D S . k+1, n, N)$ for a pair of fully interactive cubes $\mathbf{C}_{L_{k+1}}^{(n)}(\mathbf{x}) n$ and $\mathbf{C}_{L_{k+1}}^{(n)}(\mathbf{y})$. We adapt to the continuum a very crucial and hard result obtained in the paper [2] and which generalized to multiparticle systems some previous work by von Dreifus and Klein [13] on the lattice and Stollmann [12] in the continuum for single particle models.

Lemma 26. Let $J=\kappa(n)+5$ with $\kappa(n)=n^{n}$ and $E \in \mathbb{R}$. Suppose that

(i) $\mathbf{C}_{L_{k+1}}^{(n)}(\mathbf{x})$ is E-CNR.

(ii) $M\left(\mathbf{C}_{L_{k+1}}^{(n)}(\mathbf{x}), E\right) \leq J$.

Then there exists $\widetilde{L}_{2}^{*}(J, N, d) \geq 0$ such that if $L_{0} \geq \widetilde{L}_{2}^{*}(J, N, d)$ we have that $\mathbf{C}_{L_{k+1}}^{(n)}(\mathbf{x})$ is $(E, m)-N S$.

Proof. Since $M\left(\mathbf{C}_{L_{k+1}}^{(n)}(\mathbf{X}), E\right) \leq J$, there exist at most $J$ cubes of side length $2 L_{k}$ contained in $\mathbf{C}_{L_{k+1}}^{(n)}(\mathbf{x})$ that are $(E, m)$ -S with centers at distance $\geq 7 N L_{k}$. Therefore, we can find $\mathbf{x}_{i} \in \mathbf{C}_{L_{k+1}}^{(n)}(\mathbf{x}) \cap \Gamma_{\mathbf{x}}$ with $\Gamma_{\mathbf{x}}=\mathbf{x}+\left(L_{k} / 3\right) \mathbb{Z}^{n d}$.

$$
\operatorname{dist}\left(\mathbf{x}_{i}, \partial \mathbf{C}_{L_{k+1}}^{(n)}(\mathbf{x})\right) \geq 2 L_{k}, \quad i=1, \ldots, r \leq J
$$

such that, if $\mathbf{x}_{0} \in \mathbf{C}_{L_{k+1}}^{(n)}(\mathbf{x}) \backslash \bigcup_{i=1}^{r} \mathbf{C}_{2 L_{k}}^{(n)}\left(\mathbf{x}_{i}\right)$, then the cube $\mathbf{C}_{L_{k}}^{(n)}\left(\mathbf{x}_{0}\right)$ is $(E, m)$-NS.

We do an induction procedure in $\mathbf{C}_{L_{k+1}}^{(n, i n t)}(\mathbf{x})$ and start with $\mathbf{x}_{0} \in \mathbf{C}_{L_{k+1}}^{(n, \text { int })}(\mathbf{x})$. We estimate $\left\|\mathbf{1}_{\mathbf{C}_{L_{k+1}}^{(n, o u t)}(\mathbf{x})} \mathbf{G}_{L_{k+1}}^{(n)}(E) \mathbf{1}_{\mathbf{C}_{L_{k}}^{(n, i n t)}\left(\mathbf{x}_{0}\right)}\right\|$. Suppose that $\mathbf{x}_{0}, \ldots, \mathbf{x}_{\ell}$ have been choosen for $\ell \geq 0$, we have two cases:

Case $(a) \cdot \mathbf{C}_{L_{k}}^{(n)}\left(\mathbf{x}_{\ell}\right)$ is $(E, m)$-NS.

In this case, we apply the (GRI) Theorem 9 and obtain

$$
\begin{aligned}
& \left\|\mathbf{1}_{\mathbf{C}_{L_{k+1}}^{(n, o u t)}(\mathbf{x})} \mathbf{G}_{\mathbf{C}_{L_{k+1}}^{(n)}(\mathbf{x})}^{(n)}(E) \mathbf{1}_{\mathbf{C}_{L_{k+1}}^{(n, \text { int })}\left(\mathbf{x}_{0}\right)}\right\| \\
& \leq C_{\text {geom }}\left\|\mathbf{1}_{\mathbf{C}_{\left.L_{k+1}^{(n, o u t}\right)}^{(\mathbf{x})}} \mathbf{G}_{\mathbf{C}_{L_{k+1}}^{(n)}(\mathbf{x})}^{(n)}(E) \mathbf{1}_{\mathbf{C}_{L_{k+1}^{(n, o u t)}}^{\left(\mathbf{x}_{0}\right)}}\right\| \\
& \cdot\left\|\mathbf{1}_{\mathbf{C}_{L_{k+1}}^{(n, o u t)}(\mathbf{x})} \mathbf{G}_{\mathbf{C}_{L_{k+1}}^{(n)}\left(\mathbf{x}_{0}\right)}^{(n)}(E) \mathbf{1}_{\mathbf{C}_{L_{k+1}}^{(n, i n t)}\left(\mathbf{x}_{0}\right)}\right\| \\
& \leq C_{\text {geom }}\left\|\mathbf{1}_{\mathbf{C}_{L_{k+1}}^{(n, o u t)}(\mathbf{x})} \mathbf{G}_{\mathbf{C}_{L_{k+1}}^{(n)}(\mathbf{x})}^{(n)}(E) \mathbf{1}_{\mathbf{C}_{L_{k+1}}^{(n, o u t)}(\mathbf{x})}\right\| \cdot \mathrm{e}^{-\gamma\left(m, L_{k}, n\right) L_{k}} .
\end{aligned}
$$

We replace in the above analysis $\mathbf{x}$ with $\mathbf{x}_{\ell}$ and we get

$$
\begin{aligned}
& \left\|1_{\mathbf{C}_{L_{k+1}}^{(n, o u t)}\left(\mathbf{x}_{\ell}\right)} \mathbf{G}_{\mathbf{C}_{L_{k+1}}^{(n)}\left(\mathbf{x}_{\ell}\right)}^{(n)}(E) \mathbf{1}_{\mathbf{C}_{L_{k+1}}^{(n, i n t)}\left(\mathbf{x}_{\ell}\right)}\right\| \\
& \quad \leq 3^{n d}\left\|\mathbf{1}_{\mathbf{C}_{L_{k+1}}^{(n, o u t)}\left(\mathbf{x}_{\ell}\right)} \mathbf{G}_{\mathbf{C}_{L_{k+1}}^{(n)}\left(\mathbf{x}_{\ell}\right)}^{(n)}(E) \mathbf{1}_{\mathbf{C}_{L_{k+1}}^{(n, i n t)}\left(\mathbf{x}_{\ell+1}\right)}\right\|,
\end{aligned}
$$

where $\mathbf{x}_{\ell+1}$ is choosen in such a way that the norm in the right hand side in the above equation is maximal. Observe that $\left|\mathbf{x}_{\ell}-\mathbf{x}_{\ell+1}\right|=L_{k} / 3$. We therefore obtain

$$
\begin{aligned}
& \left\|\mathbf{1}_{\mathbf{C}_{L_{k+1}}^{(n, o u t)}(\mathbf{x})} \mathbf{G}_{\mathbf{C}_{L_{k+1}}^{(n)}\left(\mathbf{x}_{\ell}\right)}^{(n)}(E) \mathbf{1}_{\mathbf{C}_{L_{k+1}}^{(n, i n t)}\left(\mathbf{x}_{\ell}\right)}\right\| \\
& \quad \leq C_{g e o m} 3^{n d} \mathrm{e}^{-\gamma\left(m, L_{k}, n\right) L_{k}}\left\|\mathbf{1}_{\mathbf{C}_{L_{k+1}}^{(n, o u t)}(\mathbf{x})} \mathbf{G}_{\mathbf{C}_{L_{k+1}}^{(n)}(\mathbf{x})}^{(n)}(E) \mathbf{1}_{\mathbf{C}_{L_{k+1}}^{(n, i n t)}\left(\mathbf{x}_{\ell+1}\right)}\right\| \\
& \quad \leq \delta_{+} \| \mathbf{1}_{\mathbf{C}_{L_{k+1}}^{(n, o u t)}(\mathbf{x})} \mathbf{G}_{\mathbf{C}_{L_{k+1}}^{(n)}(\mathbf{x})}^{(n)}(E) \mathbf{1}_{\mathbf{C}_{L_{k+1}}^{(n, \text { nint })}\left(\mathbf{x}_{\ell+1}\right)}, \\
& \text { with } \delta_{+}=3^{\text {nd }} C_{\text {geom }} \mathrm{e}^{-\gamma\left(m, L_{k}, n\right) L_{k}} .
\end{aligned}
$$

Case (b). $\mathbf{C}_{L_{k}}^{(n)}\left(\mathbf{x}_{\ell}\right)$ is $(E, m)-\mathrm{S}$. Thus, there exists $i_{0}=1, \ldots, r$ such that $\mathbf{C}_{L_{k}}^{(n)}\left(\mathbf{x}_{\ell}\right) \subset \mathbf{C}_{2 L_{k}}^{(n)}\left(\mathbf{x}_{i_{0}}\right)$. We apply again the (GRI) this time with $\mathbf{C}_{L_{k+1}}^{(n)}(\mathbf{x})$ and $\mathbf{C}_{2 L_{k}}^{(n)}\left(\mathbf{x}_{i_{0}}\right)$ and obtain

$$
\begin{aligned}
& \left\|\mathbf{1}_{\mathbf{C}_{L_{k+1}}^{(n, \text { out })}(\mathbf{x})} \mathbf{G}_{\mathbf{C}_{L_{k+1}}^{(n)}(\mathbf{x})}^{(n)}(E) \mathbf{1}_{\mathbf{C}_{2 L_{k}}^{(n, \text { nint })}\left(\mathbf{x}_{i_{0}}\right)}\right\|
\end{aligned}
$$

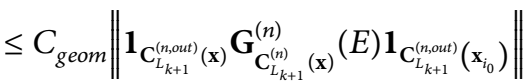

$$
\begin{aligned}
& \times\left\|\mathbf{1}_{\mathbf{C}_{L_{k}}^{(n, \text { out })}\left(\mathbf{x}_{i_{0}}\right)} \mathbf{G}_{\mathbf{C}_{L_{k}}^{(n)}\left(\mathbf{x}_{i_{0}}\right)}^{(n)}(E) \mathbf{1}_{\mathbf{C}_{L_{k}}^{(n, i n t)}\left(\mathbf{x}_{i_{0}}\right) \|}\right\| \\
& \leq C_{\text {geom }} \mathrm{e}^{\left(2 L_{k}\right)^{1 / 2}} \cdot\left\|\mathbf{1}_{\mathbf{C}_{L_{k+1}}^{(n, o u t)}(\mathbf{x})} \mathbf{G}_{\mathbf{C}_{L_{k+1}}^{(n)}(\mathbf{x})}^{(E)}(E) \mathbf{1}_{\mathbf{C}_{2 L_{k}}^{(n, o u t)}\left(\mathbf{x}_{i_{0}}\right)}\right\| \text {. }
\end{aligned}
$$

We have almost everywhere

$$
\mathbf{1}_{\mathbf{C}_{2 L_{k}}^{(n, o u t)}\left(\mathbf{x}_{i_{0}}\right)} \sum_{\tilde{\mathbf{x}} \in \mathbf{C}_{2 L_{k}}^{(n)}\left(\mathbf{x}_{i_{0}}\right) \cap \Gamma_{\mathbf{x}_{i_{0}}}, \mathbf{C}_{L_{k}}^{(n)}(\widetilde{\mathbf{x}}) \not \subset \mathbf{C}_{2 L_{k}}^{(n)}\left(\mathbf{x}_{i_{0}}\right)} \mathbf{1}_{\mathbf{C}_{L_{k}}^{(n, \text { nint })}(\mathbf{x})} .
$$

Hence, by choosing $\widetilde{\mathbf{x}}$ is such a way that the right hand side is maximal, we get

$$
\begin{aligned}
& \left\|\mathbf{1}_{\mathbf{C}_{L_{k+1}}^{(n, \text { out })}(\mathbf{x})} \mathbf{G}_{\mathbf{C}_{L_{k+1}}^{(n)}(\mathbf{x})}^{(n)}(E) \mathbf{1}_{\mathbf{C}_{2 L_{k}}^{(n, \text { nint })}\left(\mathbf{x}_{i_{0}}\right)}\right\| \\
& \quad \leq 6^{\text {nd }} \cdot\left\|\mathbf{1}_{\mathbf{C}_{L_{k+1}}^{(n, \text { out })}(\mathbf{x})} \mathbf{G}_{\mathbf{C}_{L_{k+1}}^{(n)}(\mathbf{x})}^{(n)}(E) \mathbf{1}_{\mathbf{C}_{L_{k+1}}^{(n, \text { int })}\left(\mathbf{x}^{\sim}\right)}\right\| .
\end{aligned}
$$

Since $\mathbf{C}_{L_{k}}^{(n)}(\widetilde{\mathbf{x}}) \not \subset \mathbf{C}_{2 L_{k}}^{(n)}\left(\mathbf{x}_{i_{0}}\right), \widetilde{\mathbf{x}} \in \mathbf{C}_{2 L_{k}}^{(n)}\left(\mathbf{x}_{i_{0}}\right)$ and the cubes $\mathbf{C}_{2 L_{k}}^{(n)}\left(\mathbf{x}_{i}\right)$ are disjoint, we obtain that

$$
\mathbf{C}_{L_{k}}^{(n)}(\widetilde{\mathbf{x}}) \not \subset \bigcup_{i=1}^{r} \mathbf{C}_{2 L_{k}}^{(n)}\left(\mathbf{x}_{i}\right),
$$

so that the cube $\mathbf{C}_{L_{k}}^{(n)}(\widetilde{\mathbf{x}})$ must be $(E, m)$-NS. We therefore perform a new step as in case (a) and obtain

$$
\left.\ldots \leq 6^{n d} 3^{n d} C_{g e o m} \mathrm{e}^{-\gamma\left(m, L_{k}, n\right) L_{k}} \cdot \| \mathbf{1}_{\mathbf{C}_{L_{k+1}}^{(n, o u t)}(\mathbf{x})} \mathbf{G}_{\mathbf{C}_{L_{k+1}}^{(n)}(\mathbf{x})}^{(n)}(E) \mathbf{1}_{\mathbf{C}_{L_{k+1}}^{(n, i n t)}\left(\mathbf{x}_{\ell+1}\right)}\right) \| \text {, }
$$

with $\mathbf{x}_{\ell+1} \in \Gamma_{\mathbf{x}}$ and $\left|\widetilde{\mathbf{x}}-\mathbf{x}_{\ell+1}\right|=L_{k} / 3$.

Summarizing, we get $\mathbf{x}_{\ell+1}$ with

$$
\left\|\mathbf{1}_{\mathbf{C}_{L_{k+1}}^{(n, \text { out })}(\mathbf{x})} \mathbf{G}_{\mathbf{C}_{L_{k+1}}^{(n)}(\mathbf{x})}^{(n)}(E) \mathbf{1}_{\mathbf{C}_{L_{k}}^{(n, i n t)}\left(\mathbf{x}_{\ell}\right)}\right\| \leq \delta_{0}\left\|\mathbf{1}_{\mathbf{C}_{L_{k+1}}^{(n, o u t)}(\mathbf{x})} \mathbf{G}_{\mathbf{C}_{L_{k+1}}^{(n)}(\mathbf{x})}^{(n)}(E) \mathbf{1}_{\mathbf{C}_{L_{k+1}}^{(n, i n t)}\left(\mathbf{x}_{\ell+1}\right)}\right\| \text {, }
$$


with $\delta_{0}=18^{n d} C_{\text {geom }}^{2} \mathrm{e}^{\left(2 L_{k}\right)^{1 / 2}} \mathrm{e}^{-\gamma\left(m, L_{k}, n\right) L_{k}}$. After $\ell$ iterations with $n_{+}$steps of case (a) and $n_{0}$ steps of case (b), we obtain

$$
\begin{aligned}
& \left\|\mathbf{1}_{\mathbf{C}_{L_{k+1}}^{(n, \text { out })}(\mathbf{x})} \mathbf{G}_{\mathbf{C}_{L_{k+1}}^{(n)}(\mathbf{x})}^{(n)}(E) \mathbf{1}_{\mathbf{C}_{L_{k+1}}^{(n, \text { int })}\left(\mathbf{x}_{0}\right)}\right\| \leq\left(\delta_{+}\right)^{n_{+}}\left(\delta_{0}\right)^{n_{0}} \\
& \quad \times\left\|\mathbf{1}_{\mathbf{C}_{L_{k+1}}^{(n, \text { out })}(\mathbf{x})} \mathbf{G}_{\mathbf{C}_{L_{k+1}}^{(n)}(\mathbf{x})}^{(n)}(E) \mathbf{1}_{\mathbf{C}_{L_{k}}^{(n, \text { int }}\left(\mathbf{x}_{\ell}\right)}\right\| .
\end{aligned}
$$

Now since $\gamma\left(m, L_{k}, n\right) \geq m$ we have that

$$
\delta_{+} \leq 3^{\text {nd }} \cdot C_{g e o m} \mathrm{e}^{-m L_{k}} .
$$

So $\delta_{+}$can be made arbitrarily small if $L_{0}$ and hence $L_{k}$ is large enough. We also have for $\delta_{0}$

$$
\begin{aligned}
\delta_{0}= & 18^{n d} C_{\text {geom }}^{2} \mathrm{e}^{\left(2 L_{k}\right)^{1 / 2}} \mathrm{e}^{-\gamma\left(m, L_{k}, n\right) L_{k}} \\
& \cdot 18^{n d} C_{\text {geom }}^{2} \mathrm{e}^{\sqrt{2} L_{k}^{1 / 2}} \mathrm{e}^{-\gamma\left(m, L_{k}, n\right) L_{k}} \\
\leq & 18^{n d} C_{\text {geom }}^{2} \mathrm{e}^{\sqrt{2} L_{k}^{1 / 2}-m L_{k}} \leq \frac{1}{2} .
\end{aligned}
$$

For large $L_{0}$ hence $L_{k}$. Using the (GRI), we can iterate if $\mathbf{C}_{L_{k+1}}^{(n, \text { out })}(\mathbf{x}) \cap \mathbf{C}_{L_{k}}^{(n)}\left(\mathbf{x}_{\ell}\right)=\varnothing$. Thus, we can have at least $n_{+}$steps of case (a) with

$$
\begin{aligned}
& \left\|\mathbf{1}_{\mathbf{C}_{L_{k+1}}^{(n, o u t)}(\mathbf{x})} \mathbf{G}_{\mathbf{C}_{L_{k+1}}^{(n)}(\mathbf{x})}^{(n)}(E) \mathbf{1}_{\left.\mathbf{C}_{L_{k+1}^{(n, i n t)}}^{(n)} \mathbf{x}\right)}\right\| \leq\left(\frac{L_{k+1}}{L_{k}}\right) \cdot \delta_{+}^{n_{+}} \cdot \mathrm{e}^{L_{k+1}^{1 / 2}} \\
& \quad \leq\left(\frac{L_{k+1}}{L_{k}}\right) \cdot\left[3^{n d} \cdot C_{g e o m} \cdot \mathrm{e}^{-\gamma\left(m, L_{k}, n\right)}\right]^{\left(L_{k+1} / L_{k}\right)-7 J} \times \mathrm{e}^{L_{k+1}^{1 / 2}} \\
& \quad \leq L_{k+1}^{n d} L_{k+1}^{-(n d / \alpha)} C(n, d)^{\left(L_{k+1} / L_{k}\right)-7 J} \mathrm{e}^{-\gamma\left(m, L_{k}, n\right)\left(\left(L_{k+1} / L_{k}\right)-7 J\right)} \times \mathrm{e}^{L_{k+1}^{1 / 2}} \\
& \quad \leq L_{k+1}^{n d / 3} \mathrm{e}^{\left(L_{k+1}^{1 / 3}-7 J\right) \ln C(n, d)} \mathrm{e}^{-\gamma\left(m, L_{k}, n\right)\left(L_{k+1}^{1 / 3}-7 J\right)} \mathrm{e}^{L_{k+1}^{1 / 2}} \\
& \quad \leq \mathrm{e}^{-\left[(-n d / 3) \ln \left(L_{k+1}\right)-L_{k+1}^{1 / 3} \ln (C)+7 J \ln (C(n, d))+\gamma\left(m, L_{k}, n\right) L_{k+1}^{1 / 3}-7 J \gamma\left(m, L_{k}, n\right)-L_{k+1}^{1 / 2}\right]} \\
& \quad \leq \mathrm{e}^{-\left[(-n d / 3)\left(\ln L_{k+1} / L_{k+1}\right)-\left(L_{k+1}^{1 / 3} \ln (C(n, d))\right) / L_{k+1}+(7 J \ln (C(n, d))) / L_{k+1}+\gamma\left(m, n, L_{k}\right)\left(L_{k+1}^{1 / 3}\right) / L_{k+1}-7 J\left(\gamma\left(m, L_{k}, n\right)\right) / L_{k+1}-L_{k+1}^{-1 / 2}\right]} \\
& \quad \leq \mathrm{e}^{-m^{\prime} L_{k+1}},
\end{aligned}
$$

where

$m^{\prime}=\frac{1}{L_{k+1}}\left[n_{+} \gamma\left(m, L_{k}, n\right) L_{k}-n_{+} \ln \left(2^{N d} N d L_{k}^{n d-1}\right)\right]-\frac{1}{L_{k+1}^{1 / 2}}$

with

$$
L_{k+1} L_{k}^{-1}-7 J \leq n_{+} \leq L_{k+1} L_{k}^{-1}
$$

we obtain

$$
\begin{aligned}
m^{\prime} \geq & \gamma\left(m, n, L_{k}\right)-\gamma\left(m, L_{k}, n\right) \frac{7 J L_{k}}{L_{k+1}} \\
& -\frac{1}{L_{k+1}} \frac{L_{k+1}}{L_{k}} \ln \left(\left(2_{N d} N d\right) L_{k}^{n d-1}\right)-\frac{1}{L_{k+1}^{1 / 2}} \\
\geq & \gamma\left(m, L_{k}, n\right)-\gamma\left(m, L_{k}, n\right) 7 J L_{k}^{-1 / 2} \\
& -L_{k}^{-1}\left(\ln \left(2^{N d} N d\right)\right)-(n d-1) \ln \left(L_{k}\right)-L_{k}^{-3 / 4} \\
\geq & \gamma\left(m, L_{k}, n\right)\left[1-\left(7 J+\ln \left(2^{N d} N d\right)+N d\right) L_{k}^{-1 / 2}\right],
\end{aligned}
$$

provided $L_{0} \geq \widetilde{L}_{2}^{*}$ for some large enough $\widetilde{L}_{2}^{*}(J, N, d) \geq 0$. Finally, we obtain that $m^{\prime} \geq \gamma\left(m, L_{k+1}, n\right)$. This proves the result.

The main result of this subsection is Theorem 28 below. We will need the following preliminary result. 
Lemma 27. Given $k \geq 0$, asssume that property (DS. $k, n, N)$ holds true for all pairs of separable FI cubes. Then for any $\ell \geq 1$

$$
\mathbb{P}\left\{M_{\mathrm{FI}}\left(\mathbf{C}_{L_{k+1}}^{(n)}(\mathbf{u}), I\right) \geq 2 \ell\right\} \leq C(n, N, d, \ell) L_{k}^{2 \ell d n \alpha} L_{k}^{-2 \ell \ell 4^{N-n}}
$$

Proof. See the proof in Appendix F.

Theorem 28. Let $1 \leq n \leq N$. There exists $L_{2}^{*}=L_{2}^{*}(N, d) \geq 0$ such that if $L_{0} \geq L_{2}^{*}$ and if for $k \geq 0$

(i) (DS. $\left.k-1, n^{\prime}, N\right)$ for all $n^{\prime} \in[1, n)$ holds true,

(ii) $\left(D S . k, n^{\prime}, N\right)$ holds true for all pairs of FI cubes,

then (DS. $k+1, n, N)$ holds true for any pairs of separable FI cubes $\mathbf{C}_{L_{k+1}}^{(n)}(\mathbf{x})$ and $\mathbf{C}_{k+1}^{(n)}(\mathbf{y})$.

Above we use the convention (DS. $-1, n, N)$ means no assumption.

Proof. Consider a pair of separable FI cubes $\mathbf{C}_{L_{k+1}}^{(n)}(\mathbf{x})$ and $\mathbf{C}_{L_{k+1}}^{(n)}(\mathbf{y})$ and set $J=\kappa(n)+5$. Define

$$
\begin{aligned}
B_{k+1} & =\left\{\exists E \in I_{0}: \mathbf{C}_{\mathrm{L}_{k+1}}^{(n)}(\mathbf{x}) \text { and } \mathbf{C}_{\mathrm{L}_{k+1}}^{(n)}(\mathbf{y}) \text { are }(E, m)-\mathrm{S}\right\} \\
\Sigma & =\left\{\exists E \in I_{0}: \text { neither } \mathbf{C}_{\mathrm{L}_{k+1}}^{(n)}(\mathbf{x}) \text { nor } \mathbf{C}_{\mathrm{L}_{k+1}}^{(n)}(\mathbf{y}) \text { is } E-\mathrm{CNR}\right\} \\
S_{\mathbf{x}} & =\left\{\exists E \in I_{0}: M\left(\mathbf{C}_{\mathrm{L}_{k+1}}^{(n)}(\mathbf{x}) ; E\right) \geq J+1\right\} \\
S_{\mathbf{y}} & =\left\{\exists E \in I_{0}: M\left(\mathbf{C}_{\mathrm{L}_{k+1}}^{(n)}(\mathbf{y}) ; E\right) \geq J+1\right\} .
\end{aligned}
$$

Let $\omega \in B_{k+1}$. If $\omega \notin \Sigma \cup \mathrm{S}_{\mathbf{x}}$, then $\forall E \in I_{0}$ either $\mathbf{C}_{L k+1}^{(n)}(\mathbf{x})$ or $\mathbf{C}_{L_{k+1}}^{(n)}(\mathbf{y})$ is $E$-CNR and $M\left(\mathbf{C}_{L_{k+1}}^{(n)}(\mathbf{x}), E\right) \leq J$. The cube $\mathbf{C}_{L_{k+1}}^{(n)}(\mathbf{x})$ cannot be $E$-CNR: indeed, by Lemma 26 it would be $(E, m)$ NS. So the cube $\mathbf{C}_{L_{k+1}}^{(n)}(\mathbf{y})$ is $E$-CNR and $(E, m)$-S. This implies again by Lemma 26 that

$$
M\left(\mathbf{C}_{L_{k+1}}^{(n)}(\mathbf{y}), E\right) \geq J+1 .
$$

Therefore, $\omega \in S_{\mathbf{y}}$, so that $B_{k+1} \subset \Sigma \cup S_{\mathbf{x}} \cup S_{\mathbf{y}}$, hence

$$
\mathbb{P}\left\{B_{k+1}\right\} \leq \mathbb{P}\{\Sigma\}+\mathbb{P}\left\{S_{\mathbf{x}}\right\}+\mathbb{P}\left\{S_{\mathbf{y}}\right\},
$$

and $\mathbb{P}\{\Sigma\} \leq L_{k+1}^{-4^{N} p}$ By Theorem 7. Now let us estimate $\mathbb{P}\left\{S_{\mathbf{x}}\right\}$ and similarly $\mathbb{P}\left\{S_{\mathrm{y}}\right\}$. Since

$$
M_{\mathrm{PI}}\left(\mathbf{C}_{L_{k+1}}^{(n)}(\mathbf{x}), E\right)+M_{\mathrm{FI}}\left(\mathbf{C}_{L_{k+1}}^{(n)}(\mathbf{x}), E\right) \geq M\left(\mathbf{C}_{L_{k+1}}^{(n)}(\mathbf{x}), E\right),
$$

the inequality $M\left(\mathbf{C}_{L_{k+1}}^{(n)}(\mathbf{x}), E\right) \geq \kappa(n)+6$ implies that either $M_{\mathrm{PI}}\left(\mathbf{C}_{L_{k+1}}^{(n)}(\mathbf{x}), E\right) \geq \kappa(n)+2 \quad$ or, $\quad M_{\mathrm{FI}}\left(\mathbf{C}_{L_{k+1}}^{(n)}(\mathbf{x}), E\right) \geq 4$. Therefore, by Lemmas 25 and 27 with $(\ell=2)$,

$$
\begin{aligned}
\mathbb{P}\left\{S_{\mathbf{x}}\right\} \leq & \mathbb{P}\left\{\exists E \in I: M_{\mathrm{PI}}\left(\mathbf{C}_{L_{k+1}^{(n)}}^{(\mathbf{x})}, E\right) \geq \kappa(n)+2\right\} \\
& +\mathbb{P}\left\{\exists E \in I: M_{\mathrm{FI}}\left(\mathbf{C}_{L_{k+1}}^{(n)}(\mathbf{x}), E\right) \geq 4\right\} \\
\leq & \frac{3^{2 n d}}{2} L_{k+1}^{2 n d}\left(L_{k}^{-4^{N} p}+L_{k}^{-4^{N} p 4^{N-n}}\right) \\
& +C^{\prime}(n, N, d) L_{k+1}^{4 d n-(4 p / \alpha) 4^{N-n}} \\
\leq & C^{\prime \prime}(n, N, d)\left(L_{k+1}^{-\left(4^{N} p / \alpha\right)+2 n d}\right. \\
& \left.+L_{k+1}^{-(4 p / \alpha) 4^{N-n}+2 n d}+L_{k+1}^{-(4 p / \alpha) 4^{N-n}+4 n d}\right) \\
\leq & C^{\prime \prime \prime}(n, N, d) L_{k+1}^{-(4 p / \alpha) 4^{N-n}+4 n d} \\
\leq & \frac{1}{4} L_{k+1}^{-2 p 4^{N-n}},
\end{aligned}
$$

where we used that $\alpha=3 / 2, p \geq 4 \alpha N d=6 N d$. Finally

$$
\mathbb{P}\left\{B_{k+1}\right\} \leq L_{k+1}^{-4^{N} p}+\frac{1}{2} L_{k+1}^{-2 p 4^{N-n}} \leq L_{k+1}^{-2 p 4^{N-n}} .
$$

5.3. Mixed Pairs of Cubes. Finally, it remains only to derive $(D S . k+1, n, N)$ in case (III), i.e., for pairs of $n$-particle cubes where one is PI while the other is FI.

Theorem 29. Let $1 \leq n \leq N$. There exists $L_{3}^{*}=L_{3}^{*}(N, d) \geq 0$ such that if $L_{0} \geq L_{3}^{*}(N, d)$ and iffor $k \geq 0$

(i) $\left(\right.$ DS. $\left.k-1, n^{\prime}, N\right)$ holds true all $n^{\prime} \in[1, n)$,

(ii) $\left(D S . k, n^{\prime}, N\right)$ holds true for all $n^{\prime} \in[1, n)$,

(iii) (DS. $k, n, N)$ holds true for all pairs of FI cubes,

then (DS. $k+1, n, N)$ holds true for any pair of separable cubes $\mathbf{C}_{L_{k+1}}^{(n)}(\mathbf{x})$ and $\mathbf{C}_{L_{k+1}}^{(n)}(\mathbf{y})$ where one is PI while the other is FI.

Proof. Consider a pair of separable $n$-particle cubes $\mathbf{C}_{L_{k+1}}^{(n)}(\mathbf{x})$, $\mathbf{C}_{L_{k+1}}^{(n)}(\mathbf{y})$ and suppose that $\mathbf{C}_{L_{k+1}}^{(n)}(\mathbf{x})$ is PI while $\mathbf{C}_{L_{k+1}}^{(n)}(\mathbf{y})$ is FI. Set $J=\kappa(n)+5$ and introduce the events

$$
\begin{aligned}
B_{k+1} & =\left\{\exists E \in I_{0}: \mathbf{C}_{\mathrm{L}_{k+1}}^{(n)}(\mathbf{x}) \text { and } \mathbf{C}_{\mathrm{L}_{k+1}}^{(n)}(\mathbf{y}) \text { are }(E, m)-\mathrm{S}\right\} \\
\Sigma & =\left\{\exists E \in I_{0}: \text { neither } \mathbf{C}_{\mathrm{I}_{k+1}}^{(n)}(\mathbf{x}) \text { nor } \mathbf{C}_{\mathrm{L}_{k+1}}^{(n)}(\mathbf{y}) \text { is } E-\mathrm{CNR}\right\} \\
T_{\mathbf{x}} & =\left\{\mathbf{C}_{\mathrm{L}_{k+1}}^{(n)}(\mathbf{x}) \text { is }(E, m)-\mathrm{T}\right\} \\
S_{\mathbf{y}} & =\left\{\exists \mathrm{E} \in I_{0}: M\left(\mathbf{C}_{\mathrm{I}_{k+1}}^{(n)}(\mathbf{y}), E\right) \geq J+1\right\} .
\end{aligned}
$$

Let $\omega \in B_{k+1} \backslash\left(\Sigma \cup T_{\mathbf{x}}\right)$ then, for all $E \in I_{0}$ either $\mathbf{C}_{L_{k+1}}^{(n)}(\mathbf{x})$ is $E$ CNR or $\mathbf{C}_{L_{k+1}}^{(n)}(\mathbf{y})$ is $E$-CNR and $\mathbf{C}_{L_{k+1}}^{(n)}(\mathbf{x})$ is $\left.E, m\right)$-NT. The cube $\mathbf{C}_{L_{k+1}}^{(n)}(\mathbf{x})$ cannot be $E$-CNR. Indeed by Lemma 21 it would have been $(E, m)$-NS. Thus the cube $\mathbf{C}_{L_{k+1}}^{(n)}(\mathbf{y})$ is $E$-CNR, so by Lemma $26 M\left(\mathbf{C}_{L_{k+1}}^{(n)}(\mathbf{y}), E\right) \geq J+1$ : otherwise $\mathbf{C}_{L_{k+1}}^{(n)}(\mathbf{y})$ would be $(E, m)$-NS. Therefore, $\omega \in \mathrm{S}_{\mathbf{y}}$. Consequently,

$$
B_{k+1} \subset \Sigma \cup T_{\mathbf{x}} \cup S_{\mathbf{y}} .
$$

Recall that the probabilities $\mathbb{P}\left\{T_{\mathrm{x}}\right\}$ and $\mathbb{P}\left\{S_{\mathrm{y}}\right\}$ have already been estimated in Sections 5.1 and 5.2. We therefore obtain

$$
\begin{aligned}
& \qquad \begin{aligned}
\mathbb{P}\left\{B_{k+1}\right\} & \leq \mathbb{P}\left\{T_{\mathbf{x}}\right\}+\mathbb{P}\left\{S_{\mathbf{y}}\right\} \\
& \leq L_{k+1}^{-4^{N} p}+\frac{1}{2} L_{k+1}^{-4 p 4^{N-n}}+\frac{1}{4} L_{k+1}^{-2 p 4^{N-n}} \leq L_{k+1}^{-2 p 4^{N-n}} \cdot
\end{aligned} \\
& \text { 6. Conclusion: The Multiparticle Multiscale } \\
& \text { Analysis }
\end{aligned}
$$

\section{Conclusion: The Multiparticle Multiscale Analysis}

Theorem 30. Let $\quad 1 \leq n \leq N \quad$ and $\quad \mathbf{H}^{(n)}(\omega)=-\Delta+$ $\sum_{j=1}^{n} V\left(x_{j}, \omega\right)+\mathbf{U}$, where $\mathbf{U} . V$ satisfy (I) and (P) respectively. There exists a positive $m$ such that for any $p \geq 6 \mathrm{Nd}$ property $(D S . k, n, N)$ holds true for all $k \geq 0$ provided $L_{0}$ is large enough.

Proof. We prove that for each $n=1, \ldots, N$, property $(D S . k, n, N)$ is valid. To do so, we use an induction on the number of particles $n^{\prime}=1, \ldots, n$. For $n=1$ the property holds true for all $k \geq 0$ by the single-particle localization 
theory [12]. Now suppose that for all $n^{\prime} \in[1, n)\left(D S . k, n^{\prime}, N\right)$ holds true for all $k \geq 0$, we aim to prove that $(D S . k, n, N)$ holds true for all $k \geq 0$. For $k=0$, the property is valid using Theorem 13. Next, suppose that $\left(D S . k^{\prime}, n, N\right)$ holds true for all $k^{\prime} \in(0, k)$, then by combining this last assumption with $\left(D S . k, n^{\prime}, N\right)$ above, one can conclude that:

(i) (DS. $k, n, N)$ holds true for all $k \geq 0$ and for all pairs of PI cubes using Theorem 23.

(ii) (DS. $k, n, N)$ holds true for all $k \geq 0$ and for all pairs of FI cubes using Theorem 28.

(iii) (DS. $k, n, N)$ holds true for all $k \geq 0$ and for all pairs of MI cubes using Theorem 29.

Hence, Theorem 30 is proven.

\section{Proofs of the Results}

7.1. Proof of Theorem 1. Let $1 \leq n \leq N$. We aim to prove $\sigma\left(\mathbf{H}^{(n)}(\omega)\right)=[0,+\infty)$ almost surely. Assumption (I) implies that $\mathbf{U}$ is nonnegative and assumption $(\mathbf{P})$ also implies that $\mathbf{V}$ is nonnegative. Since, $-\Delta \geq 0$, we get that almost surely $\sigma\left(\mathbf{H}^{(n)}(\omega)\right) \subset[0,+\infty)$. It remains te see that $[0,+\infty) \subset \sigma\left(\mathbf{H}^{(n)}(\omega)\right)$ almost surely.

Let $k, m \in \mathbb{N}$. Define,

$$
B_{k, m}:=\left\{\mathbf{x} \in \mathbb{Z}^{n d}: \min _{i \neq j}\left|x_{i}-x_{j}\right| \geq r_{0}+2 k m\right\},
$$

where $r_{0}$ positive is the range of the interaction $\mathbf{U}$. We also define the following sequence in $\mathbb{Z}^{\text {nd }}$,

$$
\mathbf{x}^{k, m}:=C_{k, m}(1, \ldots, n d),
$$

where $C_{k, m}=r_{0}+2 k m+1$. Using the identification $\mathbb{Z}^{n d} \cong\left(\mathbb{Z}^{d}\right)^{n}$, we can also write $\mathbf{x}^{k, m}=C_{k, m}\left(x_{1}^{k, m}, \ldots, x_{n}^{k, m}\right)$ with each $x_{i}^{k, m} \in \mathbb{Z}^{d}, i=1, \ldots n$. Obviously, each term $\mathbf{x}^{k, m}$ of the sequence $\left(\mathbf{x}^{k, m}\right)_{k, m}$ belongs to $B_{k, m}$. For $j=1, \ldots, n$, set,

$$
H_{j}^{(1)}(\omega):=-\Delta+V\left(x_{j}, \omega\right) .
$$

We have that almost surely $\sigma\left(H_{j}^{(1)}(\omega)\right)=[0,+\infty)$ see for example [12]. So, if we set for $j=1, \ldots, n$

$$
\Omega_{j}=\left\{\omega \in \Omega: \sigma\left(H_{j}^{(1)}(\omega)\right)=[0,+\infty)\right\}
$$

$\mathbb{P}\left\{\Omega_{j}\right\}=1$ for all $j=1, \ldots, n$. Now put

$$
\Omega_{0}:=\bigcap_{j=1}^{n} \Omega_{j} .
$$

We also have that $\mathbb{P}\left\{\Omega_{0}\right\}=1$. Let $\omega \in \Omega_{0}$ for this $\omega$, By the Weyl criterion, there exist $n$ Weyl sequences $\left\{\left(\phi_{j}^{m}\right)_{m}: j=1, \ldots, n\right\}$ related to 0 and each operator $H_{j}^{(1)}(\omega)$. By the density property of compactly supported functions $C_{c}^{\infty}\left(\mathbb{R}^{d}\right)$, in $L^{2}\left(\mathbb{R}^{d}\right)$, we can directly assume that each $\phi_{j}^{m}$ is of compact support, i.e., supp $\phi_{j}^{m} \subset C_{k_{i} m}^{(1)}(0)$ for some integer $k_{j}$ large enough. Set

$$
k_{0}=\max _{j=1, \ldots, n} k_{j}
$$

and put, $\mathbf{x}^{k_{0}, m}=\left(x_{1}^{k_{0}, m}, \ldots, x_{n}^{k_{0}, m}\right) \in B_{k_{0}, m}$. We translate each function $\phi_{j}^{m}$ to have support contained in the cube $C_{k_{0} m}^{(1)}\left(x_{j}^{k_{0}}\right)$. Next consider the sequence $\left(\phi^{m} m\right)_{m}$ defined by the tensor product,

$$
\phi^{m}:=\phi_{1}^{m} \otimes \cdots \otimes \phi_{n}^{m} .
$$

We have that $\operatorname{supp} \phi^{m} \subset \mathbf{C}_{k_{0}, m}^{(n)}\left(\mathbf{x}^{k_{0}, m}\right)$ and we aim to show that, $\left(\phi^{m}\right)_{m}$ is a Weyl sequence for $\mathbf{H}^{(n)}(\omega)$ and 0 . For any $\mathbf{y} \in \mathbb{Z}^{\text {nd }}$ :

$$
\left|\left(\mathbf{H}^{(n)}(\omega) \phi^{m}\right)(\mathbf{y})\right|=\left|\left(\mathbf{H}_{0}^{(n)}(\omega) \phi^{m}\right)\right| .
$$

Indeed, for the values of $\mathbf{y}$ inside the cube $\mathbf{C}_{k_{0} m}^{(n)}\left(\mathbf{x}^{k_{0}, m}\right)$ the interaction potential $\mathbf{U}$ vanishes and for those values outside that cube, $\phi^{m}$ equals zero too. Therefore,

$$
\begin{aligned}
\left\|\mathbf{H}^{(n)}(\omega) \phi^{m}\right\| & \leq\left\|\mathbf{H}_{0}^{(n)}(\omega) \phi^{m}\right\| \\
& \leq \sum_{j=1}^{n}\left\|\left(H_{j}^{(1)}(\omega)\right) \phi_{j}^{m}\right\|
\end{aligned}
$$

which tends to zero as $m$ tends to infinity because, for all $j=1, \ldots, n$ each $\left\|\left(H_{j}^{(1)}(\omega)\right) \phi_{i}^{m}\right\| \rightarrow 0$ as $m \rightarrow+\infty$, since $\phi_{j}^{m}$ is a Weyl sequence for $H_{j}^{(1)}(\omega)$ and 0 . This completes the proof.

7.2. Proof of Theorem 2. Using the multiparticle multiscale analysis bounds in the continuum property $(D S . k, N, N)$, we extend to multiparticle systems the strategy of Stollmann [12].

For $\mathbf{x}_{0} \in \mathbb{Z}^{N d}$ and an integer $k \geq 0$, using the notations of Lemma 6

$$
\begin{aligned}
R\left(\mathbf{x}_{0}\right) & :=\max _{1 \leq \ell \leq \kappa(N)}\left|\mathbf{x}_{0}-\mathbf{x}_{(\ell)}\right| ; \quad b_{k}\left(\mathbf{x}_{0}\right):=7 N+R\left(\mathbf{x}_{0}\right) L_{k}^{-1}, \\
M_{k}\left(\mathbf{x}_{0}\right) & :=\bigcup_{\ell=1}^{\kappa(N)} C_{7 N L_{k}}^{(N)}\left(\mathbf{x}^{(\ell)}\right)
\end{aligned}
$$

and define

$$
A_{k+1}\left(\mathbf{x}_{0}\right):=\mathbf{C}_{b b_{k+1} L_{k+1}}^{(N)}\left(\mathbf{x}_{0}\right) \backslash \mathbf{C}_{b_{k} L_{k}}^{(N)}\left(\mathbf{x}_{0}\right),
$$

where the positive parameter $b$ is to be chosen later. We can easily check that

$$
M_{k}\left(\mathbf{x}_{0}\right) \subset \mathbf{C}_{b_{k} L_{k}}^{(N)}\left(\mathbf{x}_{0}\right) .
$$

Moreover, if $\mathbf{x} \in A_{k+1}\left(\mathbf{x}_{0}\right)$, then the cubes $\mathbf{C}_{L_{k}}^{(N)}(\mathbf{x})$ and $\mathbf{C}_{L_{k}}^{(n)}\left(\mathbf{x}_{0}\right)$ are separable by Lemma 6 . Now, also define

$$
\begin{aligned}
\Omega_{k}\left(\mathbf{x}_{0}\right):= & \left\{\exists E \in I_{0} \text { and } \mathbf{x} \in A_{k+1}\left(\mathbf{x}_{0}\right)\right. \\
& \left.\cap \Gamma_{k}: \mathbf{C}_{L_{k}}^{(n)}(\mathbf{x}) \text { and } \mathbf{C}_{L_{k}}^{(n)}\left(\mathbf{x}_{0}\right) \text { are }(E, m)-S\right\},
\end{aligned}
$$

with $\Gamma_{k}:=\mathbf{x}_{0}+\left(L_{k} / 3\right) \mathbb{Z}^{N d}$. Now property $(D S . k, N, N)$ combined with the cardinality of $A_{k+1}\left(\mathbf{x}_{0}\right) \cap \Gamma_{k}$ imply

$$
\begin{aligned}
\mathbb{P}\left\{\Omega_{k}\left(\mathbf{x}_{0}\right)\right\} & \leq\left(2 b b_{k+1} L_{k+1}\right)^{N d} L_{k}^{-2 p} \\
& \leq\left(2 b b_{k+1}\right)^{N d} L_{k}^{-2 p+\alpha N d} .
\end{aligned}
$$

Since, $p \geq(\alpha N d+1) / 2$ (in fact $p \geq 6 N d$ ), we get $\sum_{k=0}^{\infty} \mathbb{P}\left\{\Omega_{k}\left(\mathbf{x}_{0}\right)\right\}$ is finite. Thus, setting 
$\Omega_{\infty}:=\left\{\forall \mathbf{x}_{0} \in \mathbb{Z}^{N d}, \Omega_{k}\left(\mathbf{x}_{0}\right)\right.$ occurs finitely many times $\}$,

by the Borel Cantelli Lemma and the countability of $\mathbb{Z}^{N d}$ we have that $\mathbb{P}\left\{\Omega_{\infty}\right\}=1$. Therefore, it suffices to pick $\omega \in \Omega_{\infty}$ and prove the exponential decay of any nonzero eigenfunction $\Psi$ of $\mathbf{H}^{(N)}(\omega)$.

Let $\boldsymbol{\Psi}$ be a polynomially bounded eigenfunction satisfying (EDI) (see Theorem 10). Let $\mathbf{x}_{0} \in \mathbb{Z}^{N d}$ with positive $\left\|1_{C_{1}^{(N)}\left(x_{0}\right)} \boldsymbol{\Psi}\right\|$ (if there is no such $\mathbf{x}_{0}$, we are done.) The cube $\mathbf{C}_{L_{k}}^{(N)}\left(\mathbf{x}_{0}\right)$ cannot be $(E, m)$-NS for infinitely many $k$. Indeed, given an integer $k \geq 0$, if $\mathbf{C}_{L_{k}}^{(N)}\left(\mathbf{x}_{0}\right)$ is $(E, m)$-NS then by (EDI) and the polynomial bound on $\Psi$, we get

$$
\begin{aligned}
\left\|\mathbf{1}_{\mathbf{C}_{1}^{(N)}\left(\mathbf{x}_{0}\right)} \boldsymbol{\Psi}\right\| & \leq \| \mathbf{1}_{\mathbf{C}_{L_{k}}^{(N, \text { out })}\left(\mathbf{x}_{0}\right)} \mathbf{G}_{\mathbf{C}_{L_{k}}^{(N)}\left(\mathbf{x}_{0}\right)}^{(N)}(E) \mathbf{1}_{\mathbf{C}_{L_{k}}^{(N, \text { int })}\left(\mathbf{x}_{0}\right)\|\cdot\| \mathbf{1}_{\mathbf{C}_{L_{k}}^{(N, \text { out })}\left(\mathbf{x}_{0}\right)} \boldsymbol{\Psi} \|} \\
& \leq C\left(1+\left|\mathbf{x}_{0}\right|+L_{k}\right)^{t} \cdot \mathrm{e}^{-m L_{k}}
\end{aligned}
$$

and the last term tends to 0 as $L_{k}$ tends to infinity in contradiction with the choice of $\mathbf{x}_{0}$. So there is an integer $k_{1}=k_{1}\left(\omega, E, \mathbf{x}_{0}\right)$ finite such that $\forall k \geq k_{1}$ the cube $\mathbf{C}_{L_{k}}^{(N)}\left(\mathbf{x}_{0}\right)$ is $(E, m)-\mathrm{S}$. At the same time, since $\omega \in \Omega_{\infty}$, there exists $k_{2}=k_{2}\left(\omega, \mathbf{x}_{0}\right)$ such that if $k \geq k_{2} \Omega_{k}\left(\mathbf{x}_{0}\right)$ does not occur. We conclude that for all $k \geq \max \left\{k_{1}, k_{2}\right\}$, for all $\mathbf{x} \in A_{k+1}\left(\mathbf{x}_{0}\right) \cap \Gamma_{k}$, $\mathbf{C}_{L_{k}}^{(N)}(\mathbf{x})$ is $(E, m)$-NS. Let $\rho \in(0,1)$ and choose positive $b$ such that

$$
b \geq \frac{1+\rho}{1-\rho},
$$

so that

$$
\tilde{A}_{k+1}:=\mathbf{C}_{\left(b b_{k+1} L_{k+1}\right) / 1+\rho}^{(N)}\left(\mathbf{x}_{0}\right) \backslash \mathbf{C}_{\left(b_{k} L_{k}\right) / 1-\rho}^{(N)}\left(\mathbf{x}_{0}\right) \subset A_{k+1}\left(\mathbf{x}_{0}\right),
$$

for $\mathbf{x} \in \widetilde{A}_{k+1}\left(\mathbf{x}_{0}\right)$.

(1) Since, $\left|\mathbf{x}-\mathbf{x}_{0}\right| \geq b_{k} L_{k} /(1-\rho)$,

$$
\begin{aligned}
\operatorname{dist}\left(\mathbf{x}, \partial \mathbf{C}_{b_{k} L_{k}}^{(N)}\left(\mathbf{x}_{0}\right)\right) & \geq\left|\mathbf{x}-\mathbf{x}_{0}\right|-b_{k} L_{k} \\
& \geq\left|\mathbf{x}-\mathbf{x}_{0}\right|-(1-\rho)\left|\mathbf{x}-\mathbf{x}_{0}\right| \\
& =\rho\left|\mathbf{x}-\mathbf{x}_{0}\right|
\end{aligned}
$$

(2) Since $\left|\mathbf{x}-\mathbf{x}_{0}\right| \leq b b_{k+1} L_{k+1} / 1+\rho$,

$$
\begin{aligned}
\operatorname{dist}\left(\mathbf{x}, \partial \mathbf{C}_{b b_{k+1} L_{k+1}}^{(N)}\left(\mathbf{x}_{0}\right)\right) & \geq b b_{k+1} L_{k+1}-\left|\mathbf{x}-\mathbf{x}_{0}\right| \\
& \geq(1+\rho)\left|\mathbf{x}-\mathbf{x}_{0}\right|-\left|\mathbf{x}-\mathbf{x}_{0}\right|(7.20) \\
& =\rho\left|\mathbf{x}-\mathbf{x}_{0}\right| .
\end{aligned}
$$

Thus,

$$
\operatorname{dist}\left(\mathbf{x}, \partial A_{k+1}\left(\mathbf{x}_{0}\right)\right) \geq \rho\left|\mathbf{x}-\mathbf{x}_{0}\right| .
$$

Now, setting $k_{3}=\max \left\{k_{1}, k_{2}\right\}$, the assumption linking $b$ and $\rho$ implies that

$$
\bigcup_{k \geq k_{3}} \tilde{A}_{k+1}\left(\mathbf{x}_{0}\right)=\mathbb{R}^{N d} \backslash \mathbf{C}_{\left(b_{k_{3}} L_{k_{3}}\right) / 1-\rho}\left(\mathbf{x}_{0}\right) .
$$

Because $b b_{k+1} L_{k+1} /(1+\rho) \geq b_{k} L_{k} /(1-\rho)$. Let $k \geq k_{3}$, recall that this implies that all the cubes with centers in $A_{k+1}\left(\mathbf{x}_{0}\right) \cap \Gamma_{k}$ and side length $2 L_{k}$ are $(E, m)$-NS. Thus, for any $\mathbf{x} \in \widetilde{A}_{k+1}\left(\mathbf{x}_{0}\right)$, we choose $\mathbf{x}_{1} \in A_{k+1}\left(\mathbf{x}_{0}\right)$ such that $\mathbf{x} \in \mathbf{C}_{L_{k}}^{(n)}\left(\mathbf{x}_{1}\right)$. Therefore,

$$
\begin{aligned}
\left\|\mathbf{C}_{1}^{(N)}(\mathbf{x}) \Psi\right\| & \leq\left\|1_{\mathbf{C}_{L_{k}}^{(N, \text { nt })}\left(\mathbf{x}_{1}\right)} \boldsymbol{\Psi}\right\| \\
& \leq C \cdot \mathrm{e}^{-m L_{k}}\|\cdot\| 1_{\mathbf{C}_{L_{k}}^{(N, \text { out })}\left(\mathbf{x}_{1}\right)} \boldsymbol{\Psi} \| .
\end{aligned}
$$

Up to a set of Lebesgue measure zero, we can cover $\mathbf{C}_{L_{k}}^{(N, \text { out })}\left(\mathbf{x}_{1}\right)$ by at most $3^{N d}$ cubes

$$
\mathbf{C}_{L_{k}}^{(N, i n t)}(\widetilde{\mathbf{x}}), \quad \widetilde{\mathbf{x}} \in \Gamma_{k}, \quad\left|\widetilde{\mathbf{x}}-\mathbf{x}_{1}\right|=\frac{L_{k}}{3} .
$$

By choosing $\mathbf{x}_{2}$ which gives a maximal norm, we get

$$
\left\|1_{\mathrm{C}_{L_{k}}^{(N, \text { out })}\left(\mathrm{x}_{1}\right)} \Psi\right\| \leq 3^{N d} \cdot\left\|1_{\mathrm{C}_{L_{k}}^{(N, \text { int })}\left(\mathrm{x}_{2}\right)} \boldsymbol{\Psi}\right\|,
$$

so that

$$
\left\|1_{\mathbf{C}_{1}^{(N)}(\mathbf{x})} \Psi\right\| \leq 3^{N d} \cdot \mathrm{e}^{-m L_{k}} \cdot\left\|1_{\mathbf{C}_{L_{k}}^{(N, i n t)}\left(\mathbf{x}_{2}\right)} \boldsymbol{\Psi}\right\| .
$$

Thus, by an induction procedure, we find a sequence $\mathbf{x}_{1}, \mathbf{x}_{2}, \ldots, \mathbf{x}_{n}$ in $\Gamma_{k} \cap A_{k+1}\left(\mathbf{x}_{0}\right)$ with the bound

$$
\left\|1_{\mathbf{C}_{1}^{(N)}(\mathbf{x})} \Psi\right\| \leq\left(C \cdot 3^{N d} \exp \left(-m L_{k}\right)\right)^{n} \cdot\left\|1_{\mathbf{C}_{L_{k}}^{(N, \text { out })}\left(\mathbf{x}_{n}\right)} \Psi\right\| .
$$

Since $\left|\mathbf{x}_{i}-\mathbf{x}_{i+1}\right|=L_{k} / 3$ and $\operatorname{dist}\left(\mathbf{x}, \partial A_{k+1}\right) \geq \rho \cdot\left|\mathbf{x}-\mathbf{x}_{0}\right|$ we can iterate at least $\rho \cdot\left|\mathbf{x}-\mathbf{x}_{0}\right| \cdot 3 / L_{k}$ times until, we reach the boundary of $A_{k+1}\left(\mathbf{x}_{0}\right)$. Next, using the polynomial bound on $\Psi$, we obtain:

$$
\begin{aligned}
\left\|1_{\mathbf{C}_{1}^{(N)}(\mathbf{x})} \boldsymbol{\Psi}\right\| \leq & \left(C \cdot 3^{N d}\right)^{\left(3 \rho\left|\mathbf{x}-\mathbf{x}_{0}\right|\right) / L_{k}} \cdot \exp \left(-3 m \rho\left|\mathbf{x}-\mathbf{x}_{0}\right|\right) \\
& \times C\left(1+\left|\mathbf{x}_{0}\right|+b L_{k+1}\right)^{t} \cdot L_{k+1}^{N d} .
\end{aligned}
$$

We can conclude that given $\rho^{\prime}$ with $\rho^{\prime} \in(0,1)$, we can find $k_{4} \geq k_{3}$ such that if $k \geq k_{4}$, then

$$
\left\|1_{\mathbf{C}_{1}^{(N)}(\mathbf{x})} \Psi\right\| \leq \mathrm{e}^{-\rho \rho^{\prime} m\left|\mathbf{x}-\mathbf{x}_{0}\right|},
$$

if $\left|\mathbf{x}-\mathbf{x}_{0}\right| \geq b_{k_{4}} L_{k_{4}} /(1-\rho)$. This completes the proof of the exponential localization in the max-norm.

7.3. Proof of Theorem 3. For the proof of the multiparticle dynamical localization given the multiparticle multiscale analysis in the continuum, we refer to the paper by Boutet de Monvel et al. [19].

\section{Appendix}

\section{A. Proof of Lemma 6}

(A) Consider positive $L, \emptyset \neq \mathscr{B} \subset\{1, \ldots, n\}$ and $\mathbf{y} \in \mathbb{Z}^{n d} \cdot\left\{y_{j}\right\}_{j \in \mathscr{B}}$ is called an $L$-cluster if the union

$$
\bigcup_{j \in \mathscr{B}} C_{L}^{(1)}\left(y_{j}\right),
$$

cannot be decomposed into two nonempty disjoint subsets. Next, given two configurations $\mathbf{x}, \mathbf{y} \in \mathbb{Z}^{\text {nd }}$, we proceed as follows: 
(1) We decompose the vector $y$ into maximal $L$-clusters $\Gamma_{1}, \ldots,=\Gamma_{M}$ (each of diameter $\left.\leq 2 n L\right)$ with $M \leq n$.

(2) Each position $y_{i}$ corresponds to exactly one cluster $\Gamma_{j}, j=j(i) \in\{1, \ldots, M\}$.

(3) If there exists $j \in\{1, \ldots, M\}$ such that $\Gamma_{j} \cap \Pi \mathbf{C}_{L_{k}}^{(n)}(\mathbf{x})=\emptyset$, then the cubes $\mathbf{C}_{L_{k}}^{(n)}(\mathbf{y})$ and $\mathbf{C}_{L_{k}}^{(n)}(\mathbf{x})$ are separable.

(4) If (3) is wrong, then for all $k=1, \ldots, M$, $\Gamma_{k} \cap \Pi \mathbf{C}_{L}^{(n)}(\mathbf{x}) \neq \varnothing$. Thus for all $k=1, \ldots, M$, $\exists i=1, \ldots, n$ such that $\Gamma_{k} \cap C_{L}^{(1)}\left(x_{i}\right) \neq \varnothing$. Now for any $j=1, \ldots, n$ there exists $k=1, \ldots, M$ such $y_{j} \in \Gamma_{k}$. Therefore for such $k$, by hypothesis there exists $i=1, \ldots, n$ such that $\gamma_{k} \cap C_{L}^{(1)}\left(x_{i}\right) \neq \varnothing$. Next let $z \in \Gamma_{k} \cap C_{L}^{(1)}\left(x_{i}\right)$ so that $\left|z-x_{i}\right| \leq L$. We have that

$$
\begin{aligned}
\left|y_{j}-x_{i}\right| & \leq\left|y_{j}-z\right|+\left|z-x_{i}\right| \\
& \leq 2 n L-L+L=2 n L
\end{aligned}
$$

since $y_{j} \in \Gamma_{k}$.

Notice that above we have the bound $\left|y_{j}-z\right| \leq 2 n L-L$ because $y_{j}$ is a center of the $L$-cluster $\Gamma_{k}$ Hence for all $j=1, \ldots, n y_{j}$ must belong to one of the cubes $C_{2 n L}^{(1)}\left(x_{i}\right)$ for the $n$-positions $\left(y_{1}, \ldots, y_{n}\right)$. Set $\kappa(n)=n^{n}$. For any choice of at most $\kappa(n)$ possibilities; $\mathbf{y}=\left(y_{1}, \ldots, y_{n}\right)$ must belong to the cartesian product of $n$ cubes of side length $2 L$ i.e., an $n d$-dimensional cube of size $2 n L$, the assertion then follows.

(B) Set $R(\mathbf{y})=\max _{1 \leq i, j \leq n}\left|y_{i}-y_{j}\right|+5 N L$ and consider a cube $\mathbf{C}_{L}^{(n)}(\mathbf{x})$ with $|\mathbf{y}-\mathbf{x}| \geq R(\mathbf{y})$. Then there exist $i_{0} \in\{1, \ldots, n\}$ such that $\left|y_{i_{0}}-x_{i_{0}}\right| \geq R(\mathbf{y})$. Consider the maximal connected component $\Lambda_{\mathrm{x}}:=\bigcup_{i \in \mathscr{B}} C_{L}^{(1)}\left(x_{i}\right)$ of the union $\bigcup_{i} C_{L}^{(1)}\left(x_{i}\right)$ containing $x_{i_{0}}$. Its diameter is bounded by $2 n L$. We have

$$
\operatorname{dist}\left(\Lambda_{\mathbf{x}} ; \Pi \mathbf{C}_{L}^{(n)}(\mathbf{y})\right)=\min _{u, v}|u-v|,
$$

now, since

$$
\left|x_{i_{0}}-y_{i_{0}}\right| \leq\left|x_{i_{0}}-u\right|+|u-v|+\left|v-y_{i_{0}}\right| \text {, }
$$

then

$\operatorname{dist}\left(\Lambda_{\mathbf{x}}, \Pi \mathbf{C}_{L}^{(n)}(\mathbf{y})\right)=\min _{u, v}|u-v|-\operatorname{diam}\left(\Lambda_{\mathbf{x}}\right)-\max _{v, y_{i_{0}}}\left|v-y_{i_{0}}\right|$.

Recall that $\operatorname{diam}\left(\Lambda_{\mathrm{x}}\right) \leq 2 n L$ and

$$
\max _{v, y_{i_{0}}}\left|v-y_{i_{0}}\right| \leq \max _{v}\left|v-y_{j}\right|+\max _{y_{i_{0}}}\left|\mathrm{y}_{j}-y_{i_{0}}\right|
$$

for some $j=1, \ldots, n$ such that $v \in C_{L}^{(1)}\left(y_{j}\right)$. Finally, we get

$$
\operatorname{dist}\left(\Lambda_{\mathbf{x}}, \Pi \mathbf{C}_{L}^{(n)}(\mathbf{y})\right) \geq R(\mathbf{y})-\operatorname{diam}\left(\Lambda_{\mathbf{x}}\right)-(2 L+\operatorname{diam}(\Pi \mathbf{y})),
$$

and the latter quantity is strictly positive. This implies that $\mathbf{C}_{L}^{(n)}(\mathbf{x})$ is $\mathscr{B}$ separable from $\mathbf{C}_{L}^{(n)}(\mathbf{y})$.

\section{B. Proof of Lemma 16}

Set $R:=2 L+r_{0}$ and assume that $\operatorname{diam} \Pi \mathbf{u}=\max _{i, j}\left|u_{i}-u_{j}\right| \geq n R$. If the union of cubes $C_{R / 2}^{(1)}\left(u_{i}\right), i=1, \ldots, n$ were not decomposable into two (or more) disjoint groups, then, it would be connected hence its diameter would be bounded by $n(2(R / 2))=n R$ hence $\operatorname{diam} \Pi \mathbf{u} \leq n R$ which contradicts the hypothesis. Therefore, there exists an index subset $\mathscr{B} \subset\{1, \ldots, n\}$ such that $\left|u_{j_{1}}-u_{j_{2}}\right| \geq 2(R / 2)$ for all $j_{1} \in \mathscr{B}$ and $j_{2} \in \mathscr{B}^{c}$, this implies that

$$
\begin{aligned}
\operatorname{dist} & \left(\Pi_{\mathscr{B}} \mathbf{C}_{L}^{(n)}(\mathbf{u}), \Pi_{\mathscr{B}} \mathbf{C}_{L}^{(n)}(\mathbf{u})\right) \\
= & \min _{j_{1} \in \mathscr{B}, j_{2} \in \mathscr{B}^{c}} \operatorname{dist}\left(C_{L}^{(1)}\left(u_{j_{1}}\right), C_{L}^{(1)}\left(u_{j_{2}}\right)\right) \\
\geq & \min _{j_{1} \in \mathscr{B}, j_{2} \in \mathscr{B}^{c}}\left|u_{j_{1}}-u_{j_{2}}\right|-2 L \geq r_{0} .
\end{aligned}
$$

\section{Proof of Lemma 17}

If for some positive $R$

$$
R \leq|\mathbf{x}-\mathbf{y}|=\max _{1 \leq j \leq n}\left|x_{j}-y_{j}\right|,
$$

then there exists $1 \leq j_{0} \leq n$ such that $\left|x_{j_{0}}-y_{j_{0}}\right| \geq R$. Since both cubes are fully interactive,

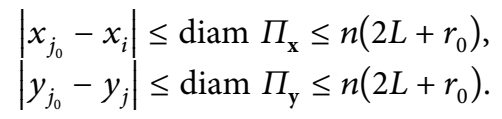

By the triangle inequality, for any $1 \leq i, j \leq n$, and $R \geq 7 n L \geq 6 n L+2 n r_{0}$, we have

$$
\begin{gathered}
\left|x_{i}-y_{j}\right| \geq\left|x_{j_{0}}-y_{j_{0}}\right|-\left|x_{j_{0}}-x_{i}\right|-\left|y_{j_{0}}-y_{j}\right| \\
\geq 6 n L+2 n r_{0}-2 n\left(2 L+r_{0}\right)=2 n L .
\end{gathered}
$$

Therefore, for any $1 \leq i, j \leq n$,

$\min _{i, j} \operatorname{dist}\left(C_{L}^{(1)}\left(x_{i}\right), C_{L}^{(1)}\left(y_{j}\right)\right) \geq \min _{i, j}\left|x_{i}-y_{j}\right|-2 L \geq 2(n-1) L$.

which proves the claim.

\section{Proof of Lemma 24}

Assume that $M^{\mathrm{sep}}\left(\mathbf{C}_{L_{k+1}}^{(n)}(\mathbf{u}), E\right)$ is less than 2 (i.e.,there is no pair of separable cubes of radius $L_{k}$ in $\left(\mathbf{C}_{L_{k+1}}^{(n)}(\mathbf{u})\right)$ but $M\left(\mathbf{C}^{(n)}(\mathbf{u}), E\right) \geq \kappa(n)+2$. Then $\mathbf{C}_{L_{k+1}}^{(n)}(\mathbf{u})$ must contain at least $\kappa(n)+2$ cubes $\mathbf{C}_{L_{k}}^{(n)}\left(\mathbf{v}_{i}\right), 0 \leq i \leq \kappa(n)+1$ which are not separable but satisfy $\left|\mathbf{v}_{i}-\mathbf{v}_{i^{\prime}}\right| \geq 7 N L_{k}$ for all $i \neq i^{\prime}$. On the other hand, by Lemma 6 there are at most $\kappa(n)$ cubes $\mathbf{C}_{2 n L_{k}}^{(n)}\left(\mathbf{y}_{i}\right)$, such that any cube $\mathbf{C}_{L_{k}}^{(n)}(\mathbf{x})$ with $\mathbf{x} \notin \bigcup_{j} \mathbf{C}_{2 n L_{k}}^{(n)}\left(\mathbf{y}_{j}\right)$, is separable from $\mathbf{C}_{L_{k}}^{(n)}\left(\mathbf{v}_{0}\right)$. Hence $\mathbf{v}_{i} \in \bigcup_{j} \mathbf{C}_{2 n L_{k}}^{(n)}\left(\mathbf{y}_{j}\right)$ for all $i=1, \ldots, \kappa(n)+1$. But since for all $i \neq i^{\prime}$ $\left|\mathbf{v}_{i}-\mathbf{v}_{i^{\prime}}\right| \geq 7 N L_{k}$ there must be at most one center $\mathbf{v}_{i}$ per cube $\mathbf{C}_{2 n L_{k}}^{(n)}\left(y_{j}\right), 1 \leq j \leq \kappa(n)$. Hence we come to a contradiction

$$
\kappa(n)+1 \leq \kappa(n) .
$$

The same analysis holds true if we consider only PI cubes. 


\section{E. Proof of Lemma 25}

Suppose that $M_{\mathrm{PI}}\left(\mathbf{C}_{L_{k+1}}^{(n)}(\mathbf{u}), I\right) \geq \kappa(n)+2$, then by Lemma 24 $M_{\mathrm{PI}}^{\text {sep }}\left(\mathbf{C}_{L_{k+1}}^{(n)}(\mathbf{u}), I\right) \geq 2$ i.e., there are at least two separable $(E, m)$ -S PI cubes $\mathbf{C}_{L_{k}}^{(n)}\left(\mathbf{u}^{\left(j_{1}\right)}\right), \mathbf{C}_{L_{k}}^{(n)}\left(\mathbf{u}^{\left(j_{2}\right)}\right)$ inside $\mathbf{C}_{L_{k+1}}^{(n)}(\mathbf{u})$. The number of possible pairs of centers $\left\{\mathbf{u}^{\left(j_{1}\right)}, \mathbf{u}^{\left(j_{2}\right)}\right\}$ such that

$$
\mathbf{C}_{L_{k}}^{(n)}\left(\mathbf{u}^{\left(j_{1}\right)}\right), \mathbf{C}_{L_{k}}^{(n)}\left(\mathbf{u}^{\left(j_{2}\right)}\right) \subset \mathbf{C}_{L_{k+1}}^{(n)}(\mathbf{u})
$$

is bounded by $\left(3^{2 n d} / 2\right) L_{k+1}^{2 n d}$. Then, setting

$$
\begin{aligned}
B_{k}= & \left\{\exists E \in I, \mathbf{C}_{L_{k}}^{(n)}\left(\mathbf{u}^{\left(j_{1}\right)}\right), \mathbf{C}_{L_{k}}^{(n)}\left(\mathbf{u}^{\left(j_{2}\right)}\right) \text { are }(E, m)-\mathrm{S}\right\} \\
& \mathbb{P}\left\{M_{\mathrm{PI}}^{\mathrm{sep}}\left(\mathbf{C}_{L_{k+1}}^{(n)}(\mathbf{u}), I\right) \geq 2\right\} \leq \frac{3^{2 n d}}{2} L_{k+1}^{2 n d} \times \mathbb{P}\left\{B_{k}\right\}
\end{aligned}
$$

with $\mathbb{P}\left\{B_{k}\right\} \leq L_{k}^{-4^{N} p}+L_{k}^{-4 p 4^{\mathrm{N}-\mathrm{n}}}$.

\section{F. Proof of Lemma 27}

Suppose there exist $2 \ell$ pairwise separable fully interactive cubes $\mathbf{C}_{L_{k}}^{(n)}\left(\mathbf{u}^{(j)}\right) \subset \mathbf{C}_{L_{k+1}}^{(n)}(\mathbf{u}), 1 \leq j \leq 2 \ell$. Then by Lemma 17 for any pair $\mathbf{C}_{L_{k}}^{(n)}\left(\mathbf{u}^{(2 i-1)}\right), \mathbf{C}_{L_{k}}^{(n)}\left(\mathbf{u}^{(2 i)}\right)$ the corresponding random Hamiltonians $\mathbf{H}_{\mathbf{C}_{L_{k}}^{(n)}\left(\mathbf{u}^{(2 i-1)}\right)}^{(n)}$ and $\mathbf{H}_{\mathbf{C}_{L_{k}}^{(n)}\left(\mathbf{u}^{(2 i)}\right)}^{(n)}$ are independent and so are their spectra and their Green functions. For $i=1, \ldots, \ell$, we consider the events:

$$
A_{i}=\left\{\exists E \in I: \mathbf{C}_{L_{k}}^{(n)}\left(\mathbf{u}^{(2 i-1)}\right) \text { and } \mathbf{C}_{L_{k}}^{(n)} \text { are }(E, m)-\mathrm{S}\right\}
$$

then by assumption $(D S . k, n, N)$, we have for $i=1, \ldots, \ell$

$$
\mathbb{P}\left\{A_{i}\right\} \leq L_{k}^{-2 p 4^{N-n}}
$$

and by independence of the events $A_{1}, \ldots, A_{\ell}$

$$
\mathbb{P}\left\{\bigcap_{1 \leq i \leq \ell} A_{i}\right\}=\prod_{i=1}^{\ell} \mathbb{P}\left\{A_{\mathrm{i}}\right\} \leq\left(L_{k}^{-2 p 4^{N-n}}\right)^{\ell} .
$$

To complete the proof, note that the total number of different families of $2 \ell$ cubes $C_{L_{k}}^{(n)}\left(\mathbf{u}^{(j)}\right) \subset \mathbf{C}_{L_{k+1}}^{(n)}(\mathbf{u}), 1 \leq j \leq 2 \ell$ is bounded by

$$
\frac{1}{(2 \ell) !}\left|\mathbf{C}_{L_{k+1}}^{(n)}(\mathbf{u})\right|^{2 \ell} \leq C(n, N, d, \ell) L_{k}^{2 \ell d n \alpha}
$$

\section{Data Availability}

No data were used to support this study.

\section{Conflicts of Interest}

The author declares that they have no conflicts of interest.

\section{References}

[1] T. Ekanga, "On two-particle Anderson localization at low energies," Comptes Rendus Mathematique, vol. 349, no. 3-4, pp. 167-170, 2011.

[2] T. Ekanga, "Localization at low energies in the multi-particle tight-binding model," 2012, https://arxiv.org/abs/1201.2339.

[3] M. Aizenmann and S. Warzel, "Localization bounds for multiparticle systems," Communication in Mathematical Physics, vol. 290, no. 3, pp. 903-934, 2009.

[4] M. Aizenmann and S. Warzel, "Complete dynamical localization in disordered quantum multi-particle systems," in XVIth International Congress on Mathematical Physics, pp. 556-565, Prague, 2009.

[5] A. Boutet de Monvel, V. Chulaevsky, P. Stollmann, and Y. Suhov, "Anderson localization for a multi-particle model with an alloy-type external random potential," https://arxiv. org/abs/1004.1300.

[6] V. Chulaevsky, "Exponential decay of eigenfunctions in a continuous muliti-particle Anderson model with subexponentially decaying interactions," 2014, https://arxiv.org/ abs/1408.4646.

[7] V. Chulaevsky and Y. Suhov, "Eigenfunctions in a twoparticle Anderson tight-binding model," Communication in Mathematical Physics, vol. 289, no. 2, pp. 701-723, 2009.

[8] V. Chulaevsky and Y. Suhov, "Multi-particle Anderson localization: induction on the number of particles," Mathematical Physics, Analysis and Geometry, vol. 12, no. 2, pp. 117-139, 2009.

[9] M. Fauser and S. Warzel, "Multiparticle localization for disordered systems on continuous space via the fractional moment method," Reviews in Mathematical Physics, vol. 27, no. 4, p. 1550010, 2015.

[10] A. Klein and S. T. Nguyen, "The boostrap multi-scale analysis for multi-particle Anderson model," Journal of Statistical Physics, vol. 151, no. 5, pp. 938-973, 2013.

[11] M. Sabri, "Anderson localization for a multi-particle quantum graph," Reviews in Mathematical Physics, vol. 26, no. 1, p. 1350020, 2014.

[12] P. Stollmann, Caught by Disorder Bounded States in Random Media, Birkhäuser Boston Inc., Boston, MA, 2001.

[13] H. von Dreifus and A. Klein, "A new proof of localization in the Anderson tight-binding model," Communication in Mathematical Physics, vol. 124, no. 2, pp. 285-299, 1989.

[14] T. Ekanga, "Multi-particle localization for weakly interacting Anderson tight-binding models," Journal of Mathematical Physics, vol. 58, no. 2, p. 043503, 2017.

[15] A. Boutet de Monvel, V. Chulaevsky, P. Stollmann, and Y. Suhov, "Wegner type-bounds for a multi-particle continuous Anderson model with an alloy-type external potential," Journal of Statistical Physics, vol. 138, no. 4-5, pp. 553-566, 2009.

[16] V. Chulaevsky and Y. Suhov, "Wegner bounds for a two-particle tight binding model," Communications in Mathematical Physics, vol. 283, no. 2, pp. 479-489, 2008.

[17] F. Germinet and A. Klein, "Operator kernel estimates for functions of generalized Schrödinger operators," Proceeding of the American Mathematical society, vol. 131, no. 3, pp. 911-920, 2002. 
[18] D. Damanik and P. Stollmann, "Multi-scale analysis implies strong dynamical localization," Geometric and Functional Analysis, vol. 11, no. 1, pp. 11-29, 2001.

[19] A. Boutet de monvel, V. Chulaevsky, and Y. Suhov, "Dynamical localization for multi-particle models with an alloy-type external random potentials," Nonlinearity, vol. 24, no. 5, pp. 1451-1472, 2011. 